\title{
Geometric study of the beta-integers for a Perron number and mathematical quasicrystals
}

\author{
par JEAN-Pierre GAZEAU et JEAN-Louis VERGER-GAUGRY
}

\begin{abstract}
RÉSUMÉ. Nous étudions géométriquement les ensembles de points de $\mathbb{R}$ obtenus par la $\beta$-numération que sont les $\beta$-entiers $\mathbb{Z}_{\beta} \subset$ $\mathbb{Z}[\beta]$ où $\beta$ est un nombre de Perron. Nous montrons qu'il existe deux schémas de coupe-et-projection canoniques associés à la $\beta$ numération, où les $\beta$-entiers se relèvent en certains points du réseau $\mathbb{Z}^{m}(m=$ degré de $\beta)$, situés autour du sous-espace propre dominant de la matrice compagnon de $\beta$. Lorsque $\beta$ est en particulier un nombre de Pisot, nous redonnons une preuve du fait que $\mathbb{Z}_{\beta}$ est un ensemble de Meyer. Dans les espaces internes les fenêtres d'acceptation canoniques sont des fractals dont l'une est le fractal de Rauzy (à quasi-homothétie près). Nous le montrons sur un exemple. Nous montrons que $\mathbb{Z}_{\beta} \cap \mathbb{R}^{+}$est de type fini sur $\mathbb{N}$, faisons le lien avec la classification de Lagarias des ensembles de Delaunay et donnons une borne supérieure effective de l'entier $q$ dans la relation : $x, y \in \mathbb{Z}_{\beta} \Longrightarrow x+y$ (respectivement $\left.x-y\right) \in$ $\beta^{-q} \mathbb{Z}_{\beta}$ lorsque $x+y$ (respectivement $x-y$ ) a un $\beta$-développement de Rényi fini.
\end{abstract}

ABSTRACT. We investigate in a geometrical way the point sets of $\mathbb{R}$ obtained by the $\beta$-numeration that are the $\beta$-integers $\mathbb{Z}_{\beta} \subset \mathbb{Z}[\beta]$ where $\beta$ is a Perron number. We show that there exist two canonical cut-and-project schemes associated with the $\beta$-numeration, allowing to lift up the $\beta$-integers to some points of the lattice $\mathbb{Z}^{m}(m=$ degree of $\beta)$ lying about the dominant eigenspace of the companion matrix of $\beta$. When $\beta$ is in particular a Pisot number, this framework gives another proof of the fact that $\mathbb{Z}_{\beta}$ is a Meyer set. In the internal spaces, the canonical acceptance windows are fractals and one of them is the Rauzy fractal (up to quasi-dilation). We show it on an example. We show that $\mathbb{Z}_{\beta} \cap \mathbb{R}^{+}$is finitely generated over $\mathbb{N}$ and make a link with the classification of Delone sets proposed by Lagarias. Finally we give an effective upper bound for the integer $q$ taking place in the relation: $x, y \in \mathbb{Z}_{\beta} \Longrightarrow x+y$ (respectively $\left.x-y\right) \in \beta^{-q} \mathbb{Z}_{\beta}$ if $x+y$ (respectively $x-y$ ) has a finite Rényi $\beta$-expansion.

Manuscrit reçu le 7 mai 2002. 


\section{Introduction}

Gazeau [Gaz], Burdik et al [Bu] have shown how to construct a discrete set $\mathbb{Z}_{\beta} \subset \mathbb{Z}[\beta] \subset \mathbb{R}$ which is a Delone set [Mo], called set of $\beta$-integers (or beta-integers), when $\beta>1$ is a Pisot number of degree greater than 2. A beta-integer has by definition no fractional part in its Rényi $\beta$ expansion $[\mathrm{Re}][\mathrm{Pa}]$. As basic feature, this Delone set is self-similar, namely $\beta \mathbb{Z}_{\beta} \subset \mathbb{Z}_{\beta}$.

Since the general notion of $\beta$-expansion of real numbers (see section 2 for definitions) was created by Rényi for any real number $\beta>1$, the set of beta-integers $\mathbb{Z}_{\beta}$, defined as the set of real numbers equal to the integer part of their $\beta$-development, is defined without ambiguity in full generality and is self-similar by construction: $\beta \mathbb{Z}_{\beta} \subset \mathbb{Z}_{\beta}$. The main questions we may address are the following: (Q1) For which $\beta>1$ is $\mathbb{Z}_{\beta}$ a Delone set ? or equivalently (Q1') for which $\beta>1$ is $\mathbb{Z}_{\beta}$ a uniformly discrete set? since the sets $\mathbb{Z}_{\beta}$ of beta-integers are always relatively dense by construction. Now Delone sets are classified into several types (see the definitions in the Appendix) so that the following question is also fundamental: (Q2) For which class of $\beta>1$ is $\mathbb{Z}_{\beta}$ a Delone set of a given type?

The uniform discretness property of $\mathbb{Z}_{\beta}$ is a crucial property which is not obtained for all real number $\beta$, but very few general results are known nowadays. Thurston has shown that it is the case when $\beta$ is a Pisot number [Th]. It is conjectured that it is also the case when $\beta$ is a Perron number. Apart from the Pisot case, many open questions remain (Bertrand-Matthis [Be4], Blanchard [B]]) and are expressed in terms of the $\beta$ - shift. Schmeling [Sc] has proved that the class $\mathrm{C}_{3}$ of real numbers $\beta>1$ such that the Renyi-expansion $d_{\beta}(1)$ of 1 in base $\beta$ contains bounded strings of zeros, but is not eventually periodic, has Hausdorff dimension 1 . For all $\beta$ in this class $\mathrm{C}_{3}$, the $\beta$-shift is specified [Bl]. It is obvious that the specification of the $\beta$-shift is equivalent to the fact that $\mathbb{Z}_{\beta}$ is uniformly discrete. So that the class $\mathrm{C}_{3}$ would contain all Perron numbers. The idea of exploring relationships between the $\beta$-shift and the algebraic properties of $\beta$ in number theory is due to A. Bertrand-Matthis [Be3]. In this direction, some results are known (Akiyama $[\mathrm{Ak}][\mathrm{Ak} 1]$ ). Parry $[\mathrm{Pa}]$ has proved that the $\beta$-shift is sofic when $\beta$ is a Pisot number. Lind [Li] conversely has shown that $\beta$ is a Perron number if the $\beta$-shift is sofic.

In section 2 we will recall some basic facts about the $\beta$-numeration and the beta-integers. In section 3 , we will establish the geometrical framework which is attached to the algebraic construction of the set of the beta-integers when $\beta$ is a Perron number in general (of degree $m \geq 2$ ). Namely, by geometric framework, we mean that we will show the existence of two cut-and-project schemes (see the definitions in the Appendix) embedded in a canonical way in the Jordan real decomposition of $\mathbb{R}^{m}$ where this 
decomposition is obtained by the action of the companion matrix of $\beta$, respectively of its adjoint, the second cut-and-project scheme being the dual of the first one. This will be done without invoking any substitution system on a finite alphabet [AI] or the theory of Perron-Frobenius [Mi]. These cut-and-project schemes will consist of an internal space which will be an hyperplane of $\mathbb{R}^{m}$ complementary to a one-dimensional line on which the set of $\beta$-integers will be set up in a natural way, together with the usual lattice $\mathbb{Z}^{m}$ in $\mathbb{R}^{m}$. The constituting irreducible subspaces of the internal spaces will appear by construction as asymptotic linear invariants. This will allow us to deduce several results when $\beta$ is a Pisot number: a minimal acceptance window in the internal space closely related to the Rauzy fractal, a geometrical proof that $\mathbb{Z}_{\beta}$ is a Meyer set, the fact that $\mathbb{Z}_{\beta}$ is finitely generated over $\mathbb{N}$. We will make a link on an example with the Rauzy fractal when the beta-integers arise from substitution systems of Pisot type (for instance Rauzy [Ra], Arnoux and Ito [AI], Messaoudi [Me] [Me1], Ito and Sano [IS], Chap. 7 in Pytheas Fogg [PF]). At this point, we should outline that the main difference with the substitutive approach is that the matrices involved may have negative coefficients (compare with the general approach of Akiyama [Ak] [Ak1]).

The additive properties of $\mathbb{Z}_{\beta}$ will be studied in section 4 by means of the canonical cut-and-project schemes when $\beta$ is a Pisot number: in A), we shall show that the elements of $\mathbb{Z}_{\beta} \cap \mathbb{R}^{+}$can be generated over $\mathbb{N}$ by elements of $\mathbb{Z}_{\beta}$ of small norm, in finite number, using truncated cones whose axis of revolution is the dominant eigenspace of the companion matrix of $\beta$ and a Lemma of Lind [Li] on semigroups; in B), we will provide a geometrical interpretation of the maximal preperiod of the $\beta$-expansion of some real numbers coming from the addition of two beta-integers, of the finite sets $T$ and $T^{\prime}$ in the relations $[\mathrm{Bu}] \mathbb{Z}_{\beta}^{+}+\mathbb{Z}_{\beta}^{+} \subset \mathbb{Z}_{\beta}^{+}+T, \mathbb{Z}_{\beta}^{+}-\mathbb{Z}_{\beta}^{+} \subset$ $\mathbb{Z}_{\beta}+T^{\prime}$ and an upper bound of the integer $q$ taking place in the relation $x, y \in \mathbb{Z}_{\beta}^{+} \Longrightarrow x \pm y \in \beta^{-q} \mathbb{Z}_{\beta}$ when $x+y$ and $x-y$ have finite $\beta$-expansions.

\section{Beta-numeration and beta-integers}

Let $\beta \in(1,+\infty) \backslash \mathbb{N}$. We will refer in the following to Rényi [Re], Parry [Pa] and Frougny [Fro] [Frol] [Bu]. For all $x \in \mathbb{R}$ we will denote by $\lfloor x\rfloor$, resp. $\{x\}=x-\lfloor x\rfloor$, the usual integer part of $x$, resp. its fractional part. Let us denote by $T(x)=\{\beta x\}$ the ergodic transformation sending $[0,1]$ into itself. For all $x \in[0,1]$, the iterates $T^{n}(x):=T\left(T^{n-1}(x)\right), n \geq$ 1 , with $T^{0}:=I d$ by convention, provide the sequence $\left(x_{-i}\right)_{i \geq 1}$ of digits, with $x_{-i}:=\left\lfloor\beta T^{i-1}(x)\right\rfloor$, in the finite alphabet $A=\{0,1, \cdots,\lfloor\beta\rfloor\}$. The element $x$ is then equal to its Rényi $\beta$-expansion $\sum_{j=1}^{+\infty} x_{-j} \beta^{-j}$ also denoted by $0 . x_{-1} x_{-2} x_{-3} \ldots$. The Rényi $\beta$-expansion of 1 will be denoted 
by $d_{\beta}(1)$. The operator $T$ on $[0,1]$ induces the shift $\sigma:\left(x_{-1}, x_{-2}, \ldots\right) \rightarrow$ $\left(x_{-2}, x_{-3}, \ldots\right)$ on the compact set $A^{\mathbb{N}}$ (with the usual product topology). The closure of the subset of $A^{\mathbb{N}}$ invariant under $\sigma$ takes the name of $\beta$-shift. The knowledge of $d_{\beta}(1)$ suffices to exhaust all the elements in the $\beta$-shift (Parry $[\mathrm{Pa}])$. For this let us define the following sequence $\left(c_{i}\right)_{i \geq 1}$ in $A^{\mathbb{N}}$ :

$$
c_{1} c_{2} c_{3} \cdots= \begin{cases}t_{1} t_{2} t_{3} \cdots & \text { if the Rényi } \beta-\text { expansion } \\ & d_{\beta}(1)=0 . t_{1} t_{2} \cdots \text { is infinite } \\ \left(t_{1} t_{2} \cdots t_{r-1}\left(t_{r}-1\right)\right)^{\omega} & \text { if } d_{\beta}(1) \text { is finite and equal } \\ & \text { to } 0 . t_{1} t_{2} \cdots t_{r},\end{cases}
$$

where ()$^{\omega}$ means that the word within ( ) is indefinitely repeated. Then the sequence $\left(y_{-i}\right)_{i \geq 1}$ in $A^{\mathbb{N}}$ is exactly the sequence of digits provided by the iterates of $y=\sum_{i=1}^{+\infty} y_{-i} \beta^{-i}$ by $T^{n}$ if and only if the following inequalities are satisfied: $\left(y_{-n}, y_{-(n+1)}, \ldots\right)<\left(c_{1}, c_{2}, c_{3}, \ldots\right)$ for all $n \geq$ 1 where " $<"$ means lexicographical smaller. These inequalities will be called conditions of Parry. We will now use finite subsets of the $\beta$-shift.

Definition 2.1. Let $\mathbb{Z}_{\beta}^{+}=\left\{x_{k} \beta^{k}+x_{k-1} \beta^{k-1}+\cdots+x_{1} \beta+x_{0} \mid x_{i} \in\right.$ $A, k \geq 0$, and $\left(x_{j}, x_{j-1}, \ldots, x_{1}, x_{0}, 0,0, \cdots\right)<\left(c_{1}, c_{2}, \cdots\right)$ for all $j, 0 \leq$ $j \leq k\}$ be the discrete subset of $\mathbb{R}^{+}$of the real numbers equal to the integer part of their Rényi $\beta$-expansion. The set $\mathbb{Z}_{\beta}=\mathbb{Z}_{\beta}^{+} \cup\left(-\mathbb{Z}_{\beta}^{+}\right)$is called the set of $\beta$ - integers.

For all $x \in \mathbb{R}^{+}$, if $x=\sum_{i=-\infty}^{p} x_{i} \beta^{i}$ with $p \geq 0$, is obtained by the greedy algorithm, then $\left(x_{i}\right)_{i \leq p}$ will satisfy the conditions of Parry. We will denote by $\operatorname{int}(x)=\sum_{i=0}^{p} x_{i} \beta^{i}$ the integer part of its Rényi $\beta$-expansion, respectively by $\operatorname{frac}(x)=\sum_{i=-\infty}^{-1} x_{i} \beta^{i}$ its fractional part. The element $1=\beta^{0}$ belongs to $\mathbb{Z}_{\beta}^{+}$.

Let us now turn to the case where $\beta$ is a positive real algebraic integer. Then there exists an irreducible polynomial $P(X)=X^{m}-$ $\sum_{i=0}^{m-1} a_{i} X^{i}, a_{i} \in \mathbb{Z}$ with $m=\operatorname{degree}(\beta)$ such that $P(\beta)=0$. Then $\beta=\sum_{i=0}^{m-1} a_{m-1-i} \beta^{-i}$. If $a_{j} \geq 0$ for all $j$ and $\left(a_{n}, a_{n+1}, \ldots\right)<$ $\left(a_{m-1}, a_{m-2}, \ldots, a_{0}, 0,0, \ldots\right)$ for all $n \leq m-2$, then the Rényi $\beta$-expansion of $\beta$ would be $\sum_{i=0}^{m-1} a_{m-1-i} \beta^{-i}$ from which we would deduce $d_{\beta}(1)=$ $\sum_{i=0}^{m-1} a_{m-1-i} \beta^{-i-1}$ as well. But the coefficients $a_{i}$ do not obey the conditions of Parry in general. More considerations on the relations between $\beta$-expansions and algebraicity can be found in [Be] [Be1] [Be2] [Be3] [Fro1] [Ak] [Ak1] [Sch]. Bertrand-Matthis [Be] and Schmidt [Sch] have proved that, when $\beta$ is a Pisot number, $x \in \mathbb{Q}(\beta)$ if and only if the Rényi $\beta$ expansion of $x$ is eventually periodic; in particular the Rényi $\beta$-expansion of any Pisot number is eventually periodic. 
Let us recall that a Perron number $\beta$, resp. a Lind number, resp. a Salem number, will be a real algebraic integer $\beta>1$ whose conjugates $\beta^{(i)}$ are of modulus strictly less than $\beta$, resp. of modulus less than $\beta$ with at least one conjugate of modulus $\beta$ [La], resp. of modulus less than 1 with at least one conjugate of modulus one. A Pisot number $\beta$ will be a real algebraic integer $\beta>1$ for which all the conjugates are in the open unit disc in the complex plane.

\section{Canonical cut-and-project schemes over $\mathbb{Z}_{\beta}$}

Assume that $\beta>1$ is a Perron number of degree $m \geq 2$, dominant root of the irreducible polynomial $P(X)=X^{m}-a_{m-1} X^{m-1}-a_{m-2} X^{m-2}-$ $\cdots-a_{1} X-a_{0}, \quad a_{i} \in \mathbb{Z}, a_{0} \neq 0$. All the elements $r \beta^{k}$ with $k \geq 1, r \in$ $\{1,2, \ldots,\lfloor\beta\rfloor\}$ are obviously in $\mathbb{Z}_{\beta}$. We are looking for asymptotic linear invariants associated with them, hence, by linearity, associated with the powers $\beta^{k}, k \geq 1$, of $\beta$, when $k$ tends to infinity. By linearity, they will be also associated to the beta-integers. Let us set up the general situation. For all $k \geq 0$, write $\beta^{k}=z_{m-1, k} \beta^{m-1}+z_{m-2, k} \beta^{m-2}+\cdots+$ $z_{1, k} \beta+z_{0, k}$, where all the integers $z_{0, k}, z_{1, k}, \cdots, z_{m-1, k}$ belong to $\mathbb{Z}$. Denote $Z_{k}={ }^{t}\left(z_{0, k} z_{1, k} z_{2, k} \ldots z_{m-1, k}\right), B=B^{(0)}={ }^{t}\left(1 \beta \beta^{2} \ldots \beta^{m-1}\right)$, $B^{(j)}={ }^{t}\left(1 \beta^{(j)} \beta^{(j)^{2}} \ldots \beta^{(j)^{m-1}}\right)$, where ${ }^{t}$ means transposition and the elements $\beta^{(j)}, j \in\{1,2, \cdots, m-1\}$, are the conjugate roots of $\beta=\beta^{(0)}$ in the minimal polynomial of $\beta$. Set

$$
\mathcal{B}_{k}=\left(\begin{array}{c}
\beta^{k} \\
\beta^{(1)^{k}} \\
\beta^{(2)^{k}} \\
\vdots \\
\beta^{(m-1)^{k}}
\end{array}\right) \quad \text { and } Q=\left(\begin{array}{ccccc}
0 & 1 & 0 & \cdots & 0 \\
0 & 0 & 1 & \cdots & 0 \\
\vdots & \vdots & & \ddots & 0 \\
0 & 0 & & \cdots & 1 \\
a_{0} & a_{1} & & \cdots & a_{m-1}
\end{array}\right)
$$

the $m \times m$ matrix with coefficients in $\mathbb{Z}$. The transposed matrix of $Q$ is denoted by ${ }^{t} Q$. It is the companion matrix of $P(X)$ (and of $\beta$ ). For all $p, k \in\{0,1, \cdots, m-1\}$, we have: $z_{p, k}=\delta_{p, k}$ the Kronecker symbol. It is obvious that, for all $k \geq 0$, we have $Z_{k+1}={ }^{t} Q Z_{k}$. Denote

$$
C=\left(\begin{array}{ccccc}
1 & \beta & \beta^{2} & \cdots & \beta^{m-1} \\
1 & \beta^{(1)} & \beta^{(1)^{2}} & \cdots & \beta^{(1)^{m-1}} \\
\vdots & \vdots & \vdots & & \vdots \\
1 & \beta^{(m-1)} & \beta^{(m-1)^{2}} & \cdots & \beta^{(m-1)^{m-1}}
\end{array}\right)
$$

the Vandermonde matrix of order $m$. We obtain $C Z_{k}=\mathcal{B}_{k}$ by the real and complex embeddings of $\mathbb{Q}[\beta]$ since all the coefficients $z_{j, k}, j \in$ $\{0,1, \cdots, m-1\}$, are integers and remain invariant under the conjugation operation. 
Theorem 3.1. If $V_{1}$ denotes the vector defined by the first column of $C^{-1}$, then the limit $\lim _{k \rightarrow+\infty}\left\|Z_{k}\right\|^{-1} Z_{k}$ exists and is equal to the unit vector $u:=\left\|V_{1}\right\|^{-1} V_{1}$. Moreover, all the components of $V_{1}$ are real and belong to the $\mathbb{Z}$ - module $\frac{\mathbb{Z}[\beta]}{\beta^{m-1} P^{\prime}(\beta)}$.

Proof. Since $P(X)$ is minimal, all the roots of $P(X)$ are distinct. Hence, the determinant of $C$ is $\prod_{i<j}\left(\beta^{(i)}-\beta^{(j)}\right)$ and is not zero. Let $C^{-1}=\left(\xi_{i j}\right)$. Then $C \cdot C^{-1}=I$, that is

(1)

$$
\xi_{1 i}+\xi_{2 i} \beta^{(j)}+\xi_{3 i} \beta^{(j)^{2}}+\cdots+\xi_{m i} \beta^{(j)^{m-1}}=\delta_{i, j+1},\left\{\begin{array}{l}
i=1,2, \ldots, m, \\
j=0,1, \ldots, m-1
\end{array}\right.
$$

On the other hand, the Lagrange interpolating polynomials associated with $\left\{\beta, \beta^{(1)}, \beta^{(2)}, \ldots, \beta^{(m-1)}\right\}$ are given by

$$
L_{s}(X)=\prod_{\substack{j=0 \\ j \neq s}}^{m-1} \frac{X-\beta^{(j)}}{\beta^{(s)}-\beta^{(j)}} \quad s=0,1, \ldots, m-1 .
$$

For $m$ arbitrary complex numbers $y_{1}, y_{2}, \cdots, y_{m}$, let us denote by $\sigma_{r}=\sigma_{r}\left(y_{1}, y_{2}, \cdots, y_{m}\right)=$

$\sum_{1 \leq i_{1} \leq i_{2} \leq \cdots \leq i_{r} \leq m} \prod_{j=1}^{r} y_{i_{j}}$ the $r$-th elementary symmetric function of the $m$ numbers $y_{1}, y_{2}, \cdots, y_{m}$. The degree of $L_{s}(X)$ is $m-1$ and $L_{s}(X)$ can be expressed as

$$
L_{s}(X)=\sum_{r=0}^{m-1}(-1)^{r} \sigma_{r}^{(s)} X^{m-r-1} / \prod_{\substack{r=0 \\ r \neq s}}^{m-1}\left(\beta^{(s)}-\beta^{(r)}\right)
$$

where $\sigma_{r}^{(s)}=\sigma_{r}\left(\beta, \beta^{(1)}, \cdots, \beta^{(s-1)}, \beta^{(s+1)}, \cdots, \beta^{(m-1)}\right)$ denotes the $r$-th elementary symmetric function of the $m-1$ numbers $\beta, \beta^{(1)}, \cdots, \beta^{(s-1)}$, $\beta^{(s+1)}, \cdots, \beta^{(m-1)}$ where $\beta^{(s)}$ is missing. Since these polynomials satisfy $L_{s}\left(\beta^{(k)}\right)=\delta_{s, k}$ for all $s, k=0,1, \cdots, m-1$, comparing with (1), we obtain, by identification of the coefficients

$$
\xi_{j i}=\frac{(-1)^{m-j} \sigma_{m-j}^{(i-1)}}{\prod_{\substack{r=0 \\ r \neq i-1}}^{m-1}\left(\beta^{(i-1)}-\beta^{(r)}\right)}=\frac{(-1)^{m-j} \sigma_{m-j}^{(i-1)}}{P^{\prime}\left(\beta^{(i-1)}\right)}
$$

for all $i, j=1,2, \cdots, m$. We have: $L_{s}(X)=\sum_{j=1}^{m} \xi_{j, s+1} X^{j-1}, s=$ $0,1, \cdots, m-1$. Now $C \cdot Z_{k}=\mathcal{B}_{k}$ for all $k \geq 0$, hence $Z_{k}=C^{-1} \cdot \mathcal{B}_{k}$. Each component $z_{i, k}, 0 \leq i \leq m-1, k \geq 0$ of $Z_{k}$ can be expressed as $z_{i, k}=$ $\sum_{j=1}^{m} \xi_{i+1, j} \beta^{(j-1)^{k}}$. Since $\beta$ is a Perron number, we have $\left|\beta^{(j)}\right|<\beta$ for all 
$j, 1 \leq j \leq m-1$. Hence, for all $j, 1 \leq j \leq m-1, \lim _{k \rightarrow+\infty}\left(\frac{\beta^{(j)}}{\beta}\right)^{k}=0$, and therefore $\lim _{k \rightarrow+\infty} \frac{z_{i, k}}{\beta^{k}}=\xi_{i+1,1}, i=0,1, \cdots, m-1$. Moreover,

$$
\lim _{k \rightarrow+\infty} \frac{\left(\sum_{i=0}^{m-1}\left|z_{i, k}\right|^{2}\right)^{1 / 2}}{\beta^{k}}=\lim _{k \rightarrow+\infty} \frac{\left\|Z_{k}\right\|}{\beta^{k}}=\sqrt{\sum_{i=0}^{m-1}\left|\xi_{i+1,1}\right|^{2}}=\left\|V_{1}\right\|
$$

hence the result. The fact that all the components of $V_{1}$ are real and belong to the $\mathbb{Z}$ - module $\mathbb{Z}[\beta] /\left(\beta^{m-1} P^{\prime}(\beta)\right)$ comes from the following more precise Proposition.

Proposition 3.1. The components $\left(\xi_{j, 1}\right)_{j=1, \ldots, m}$ of $V_{1}$ are given by the following explicit functions of the coefficients $a_{i}$ of $P(X)$ : $\xi_{j, 1}=\frac{a_{j-1} \beta^{j-1}+a_{j-2} \beta^{j-2}+\cdots+a_{1} \beta+a_{0}}{\beta^{j} P^{\prime}(\beta)}$. In particular, $\xi_{m, 1}=\frac{1}{P^{\prime}(\beta)}$.

Proof. We have $L_{0}(X)=\sum_{j=1}^{m} \xi_{j, 1} X^{j-1}$ and $P(X)=\prod_{j=0}^{m-1}(X-$ $\left.\beta^{(j)}\right)=L_{0}(X)(X-\beta) P^{\prime}(\beta)$. All the coefficients of $L_{0}(X)$ satisfy the following relations: $-\beta P^{\prime}(\beta) \xi_{1,1}=-a_{0},-\beta P^{\prime}(\beta) \xi_{2,1}+\xi_{1,1} P^{\prime}(\beta)=-a_{1}$, $-\beta P^{\prime}(\beta) \xi_{3,1}+\xi_{2,1} P^{\prime}(\beta)=-a_{2}, \cdots,-\beta P^{\prime}(\beta) \xi_{m, 1}+\xi_{m-1,1} P^{\prime}(\beta)=-a_{m-1}$, $\xi_{m, 1} P^{\prime}(\beta)=1$. Hence the result recursively from $\xi_{1,1}$ noting that $P^{\prime}(\beta) \in$ $\mathbb{R}-\{0\}$.

Theorem 3.2. Let $u_{B}:=B /\|B\|$. Then: (i) $u \cdot u_{B}=\|B\|^{-1}\left\|V_{1}\right\|^{-1}>$ 0 , (ii) the limit $\lim _{k \rightarrow+\infty} \frac{\left\|Z_{k+1}\right\|}{\left\|Z_{k}\right\|}$ exists and is equal to $\beta$, (iii) $u$ is an eigenvector of ${ }^{t} Q$ of eigenvalue $\beta$ and the eigenspace of $\mathbb{R}^{m}$ associated with the eigenvalue $\beta$ of ${ }^{t} Q$ is $\mathbb{R} u$, (iv) $u_{B}$ is an eigenvector of the adjoint matrix $\left({ }^{t} Q\right)^{*}=Q$ associated with the eigenvalue $\beta$ and for all $x \in \mathbb{C}^{m}: \lim _{k \rightarrow+\infty} \beta^{-k}\left({ }^{t} Q\right)^{k}(x)=(x \cdot B) V_{1}$.

Proof. (i) and (ii): From the relation $C \cdot C^{-1}=I d$ we deduce the equality $V_{1} \cdot B=1$. Hence $u \cdot B=\left\|V_{1}\right\|^{-1}>0$. Then, for all $k \geq 0$, ${ }^{t} Z_{k} \cdot B=\beta^{k}=\left\|Z_{k}\right\|^{t}\left(\frac{Z_{k}}{\left\|Z_{k}\right\|}-u+u\right) \cdot B>0$ which tends to infinity when $k$ tends to $+\infty$. Since $u-Z_{k} /\left\|Z_{k}\right\|$ tends to zero when $k$ goes to infinity, $\left\|Z_{k}\right\|$ behaves at infinity like $\beta^{k} /(u \cdot B)$, hence the limit; (iii): for all $k \geq 0,{ }^{t} Q(u)={ }^{t} Q\left(u-\frac{Z_{k}}{\left\|Z_{k}\right\|}+\frac{Z_{k}}{\left\|Z_{k}\right\|}\right)={ }^{t} Q\left(u-\frac{Z_{k}}{\left\|Z_{k}\right\|}\right)+$ $\frac{\left\|Z_{k+1}\right\|}{\left\|Z_{k}\right\|} \frac{Z_{k+1}}{\left\|Z_{k+1}\right\|}$. The first term is converging to zero and the second one to $\beta u$ when $k$ goes to infinity, from Theorem 3.1. Hence, the result since all the roots of $P(X)$ are distinct and the (real) eigenspace associated with $\beta$ is 1 -dimensional; (iv): it is clear that $B$ is an eigenvector of the adjoint matrix $Q$. If $h_{0}, h_{1}, \cdots, h_{m-1} \in \mathbb{C}, x=\sum_{j=0}^{m-1} h_{j} Z_{j}$, where $Z_{0}, Z_{1}, \cdots, Z_{m-1}$ is the canonical basis of $\mathbb{C}^{m}$, we have: $\beta^{-k}\left({ }^{t} Q\right)^{k}(x)=$ 
$\sum_{j=0}^{m-1} h_{j} \beta^{-k} Z_{k+j}=\sum_{j=0}^{m-1} h_{j} \beta^{j}\left(\frac{Z_{k+j}}{\beta^{k+j}}\right)$, but, from the proof of Theorem 3.1, $\lim _{k \rightarrow+\infty} \frac{Z_{k+j}}{\beta^{k+j}}=V_{1}$ and $\sum_{j=0}^{m-1} h_{j} \beta^{j}=x \cdot B$. We deduce the claim.

Let us denote by ${ }^{t} Q_{\mathbb{C}}$ the automorphism of $\mathbb{C}^{m}$ which is the complexification operator of ${ }^{t} Q$. Its adjoint $Q_{\mathbb{C}}$ obviously admits $\left\{B, B^{(1)}, B^{(2)}, \cdots\right.$ $\left.B^{(m-1)}\right\}$ as a basis of eigenvectors of respective eigenvalues $\beta, \beta^{(1)}, \beta^{(2)}, \ldots$ $\beta^{(m-1)}$. Let us specify their respective actions on $\mathbb{R}^{m}$. Let $s \geq 1$, resp. $t$, be the number of real, resp. complex (up to conjugation), embeddings of the number field $\mathbb{Q}(\beta)$. We have $m=s+2 t$. Assume that the conjugates of $\beta$ are $\beta, \beta^{(1)}, \cdots, \beta^{(s-1)}, \beta^{(s)}, \beta^{(s+1)}, \cdots, \beta^{(m-2)}=$ $\beta^{(s+2 t-2)}, \beta^{(m-1)}=\beta^{(s+2 t-1)}$ where $\beta^{(q)}$ is real if $q \leq s-1$ and $\beta^{(s+2 j)}=\overline{\beta^{(s+2 j+1)}}=\left|\beta^{(s+2 j)}\right| e^{i \theta_{j}}, j=0,1, \cdots, t-1$, is complex with non-zero imaginary part. Let us recall that $V_{1}$ denotes the vector defined by the first column of $C^{-1}$ (Theorem 3.1).

Corollary 3.3. (i) A basis of eigenvectors of ${ }^{t} Q_{\mathbb{C}}$ is given by the $m$ column vectors $\left\{W_{k}\right\}_{k=1,2, \cdots, m}$ of respective components $\xi_{j, k}=\frac{a_{j-1} \beta^{(k-1)^{j-1}}+a_{j-2} \beta^{(k-1)^{j-2}}+\cdots+a_{1} \beta^{(k-1)}+a_{0}}{\beta^{(k-1)^{j}} P^{\prime}\left(\beta^{(k-1)}\right)}$ with $j=1,2, \cdots, m$; in particular, $\xi_{m, k}=\frac{1}{P^{\prime}\left(\beta^{(k-1)}\right)}$; (ii) a real Jordan form for ${ }^{t} Q$ is given by the diagonal matrix $\operatorname{Diag}\left(\beta, \beta^{(1)}, \cdots, \beta^{(s-1)}, D_{0}, D_{1}, \cdots, D_{t-1}\right)$ in the basis of eigenvectors $\left\{V_{j}\right\}_{j=1, \cdots, m}$ with $V_{2}=W_{2}, \cdots, V_{s}=W_{s}, V_{s+2 j+1}=$ $\operatorname{Im}\left(W_{s+2 j+1}\right), V_{s+2 j+2}=\operatorname{Re}\left(W_{s+2 j+1}\right), j=0,1, \cdots, t-1$ and where the $2 \times 2$ real Jordan blocks $D_{j}$ are

$$
\left(\begin{array}{cc}
\left|\beta^{(s+2 j)}\right| \cos \theta_{j} & -\left|\beta^{(s+2 j)}\right| \sin \theta_{j} \\
\left|\beta^{(s+2 j)}\right| \sin \theta_{j} & \left|\beta^{(s+2 j)}\right| \cos \theta_{j}
\end{array}\right)
$$

(iii) a real Jordan form of the adjoint operator $\left({ }^{t} Q\right)^{*}=Q$ is given by the same diagonal matrix $\operatorname{Diag}\left(\beta, \beta^{(1)}, \cdots, \beta^{(s-1)}, D_{0}, D_{1}, \cdots, D_{t-1}\right)$ in the basis of eigenvectors $\left\{X_{j}\right\}_{j=1, \cdots, m}$ with $X_{1}=B, X_{2}=B^{(1)}, X_{3}=$ $B^{(2)}, \cdots, X_{s}=B^{(s-1)}, X_{s+2 j+1}=\operatorname{Im}\left(B^{(s+2 j)}\right), X_{s+2 j+2}=\operatorname{Re}\left(B^{(s+2 j)}\right)$, $j=0,1, \cdots, t-1$. The $t$ planes $\mathbb{R} X_{s+2 j+1}+\mathbb{R} X_{s+2 j+2}, j=0,1, \ldots, t-$ 1 are all orthogonal to $V_{1}$, and thus also to $u$.

Proof. (i): We apply, componentwise in the equation $\left({ }^{t} Q\right) V_{1}=\beta V_{1}$, the $\mathbb{Q}$ - automorphisms of $\mathbb{C}$ which are the real and complex embeddings of the number field $\mathbb{Q}(\beta)$. Since ${ }^{t} Q$ has rational entries and $V_{1}$ has its components in the $\mathbb{Z}$-module $\beta^{1-m}\left(P^{\prime}(\beta)\right)^{-1} \mathbb{Z}[\beta]$, we deduce the claim: $\left({ }^{t} Q\right) W_{j}=\beta^{(j-1)} W_{j}$ with $j=1,2, \cdots, m$ and where $W_{1}=V_{1}$; (ii): the restrictions of ${ }^{t} Q_{\mathbb{C}}$ to the (real) ${ }^{t} Q$ - invariant subspaces of $\mathbb{R}^{m}$ have no nilpotent parts since all the roots of $P(X)$ are distinct. Hence, a real Jordan form of ${ }^{t} Q$ is the one proposed with Jordan blocks which are $2 \times 2$ 
on the diagonal [HS]. (iii): in a similar way the equation $Q B=\beta B$ implies $Q B^{(j)}=\beta^{(j)} B^{(j)}$ with $j=0,1, \cdots, m-1$. Obviously $Q_{\mathbb{C}}$ and ${ }^{t} Q_{\mathbb{C}}$ have the same eigenvalues and $Q$ and ${ }^{t} Q$ the same real $2 \times 2$ Jordan blocks on the diagonal. The corresponding basis of eigenvectors is given by the vectors $X_{i}$ [HS]. The orthogonality between $V_{1}$ and the vector $X_{s+2 j+1}$, resp. $X_{s+2 j+2}, j=0,1, \ldots, t-1$, arises from the relation $C \cdot C^{-1}=I d$. We deduce the claim for the planes.

The linear invariants associated with the powers of $\beta$ are the invariant subspaces given by Corollary 3.3. Let us turn to the beta-integers. Betaintegers are particular $\mathbb{Z}$-linear combinations of powers of $\beta$. We will show how to construct the set $\mathbb{Z}_{\beta}$ using the above linear invariants, namely, the set $\mathbb{Z}_{\beta}$ will appear in a natural way on the line $\mathbb{R} u_{B}$ as image of a point set close to the expanding line $\mathbb{R} u$.

REMARK . - The conditions of Parry, used here in the context of matrices ${ }^{t} Q$ without any condition on the signs of the entries, give the same results as those obtained with the Perron-Frobenius theory (Minc [Mi]), when this one is applicable, that is when ${ }^{t} Q$ has non-negative entries: first, the dimensionality one for the dominant eigenspace of ${ }^{t} Q$; second, the equality $\lim _{k \rightarrow+\infty} \beta^{-k}\left({ }^{t} Q\right)^{k}(x)=(x \cdot B) V_{1}$, for $x \in \mathbb{C}^{m}$, in Theorem 3.2 (compare with Ruelle [Ru] p136 when ${ }^{t} Q$ has non-negative entries), and its consequences.

Theorem 3.4. Let $\pi_{B}$ be the orthogonal projection mapping of $\mathbb{R}^{m}$ onto $\mathbb{R} B$ and define $\mathcal{L}=\left\{x_{k} Z_{k}+x_{k-1} Z_{k-1}+\cdots+x_{1} Z_{1}+x_{0} Z_{0} \mid x_{i} \in A, k \geq\right.$ 0 , and $\left(x_{j}, x_{j-1}, \cdots, x_{1}, x_{0}, 0,0, \cdots\right)<\left(c_{1}, c_{2}, \cdots\right)$ for all $\left.j, 0 \leq j \leq k\right\}$ the ${ }^{t} Q$-invariant subset of $\mathbb{Z}^{m}$. Then: (i) the mapping $\sum_{j=0}^{k} x_{j} \beta^{j} \rightarrow$ $\sum_{j=0}^{k} x_{j} Z_{j}: \mathbb{Z}_{\beta}^{+} \rightarrow \mathcal{L} \quad$ (with the same coefficients $x_{j}$ ) is a bijection, (ii) the mapping $\pi_{B_{\mathbb{Z}^{m}}}$ is one-to-one onto its image $\mathbb{Z}[\beta]\|B\|^{-1} u_{B}$ : for any $k \geq 0, a_{0}, \cdots, a_{k} \in \mathbb{Z}$, we have $\pi_{B}\left(\sum_{i=0}^{k} a_{i} Z_{i}\right)=\left(\sum_{i=0}^{k} a_{i} \beta^{i}\right)\|B\|^{-1} u_{B}$ and conversely, any polynomial in $\beta$ on the line generated by $\|B\|^{-1} u_{B}$ can be uniquely lifted up to a $\mathbb{Z}$-linear combination of the vectors $Z_{i}$ with the same coefficients; in particular, $\pi_{B}(\mathcal{L})=\mathbb{Z}_{\beta}^{+}\|B\|^{-1} u_{B}$.

Proof. (i): this mapping $\mathbb{Z}_{\beta}^{+} \rightarrow \mathcal{L}$ is obviously surjective. Let us show that it is injective. Assume there exists a non-zero element $\sum_{j=0}^{k} x_{j} \beta^{j}$ in $\mathbb{Z}_{\beta}^{+}$such that $\sum_{j=0}^{k} x_{j} Z_{j}=0$. Since ${ }^{t}\left(\sum_{j=0}^{k} x_{j} Z_{j}\right) B=0=\sum_{j=0}^{k} x_{j} \beta^{j}$, this would mean that zero could be represented by a non-zero element. This is impossible by construction; (ii): for all $k \geq 0$, we have $\pi_{B}\left(Z_{k}\right)=$ $\beta^{k}\|B\|^{-1} u_{B}$, hence the result by linearity. The injectivity of $\pi_{B_{\mathbb{Z}^{m}}}$ comes from the assertion (i). 
Proposition 3.2. Let $u_{B, i}=\left\|X_{i}\right\|^{-1} X_{i} \quad$ if $i=1,2, \cdots, s, \quad u_{B, i}=$ $\left(\left\|X_{i}\right\|^{2}+\left\|X_{i+1}\right\|^{2}\right)^{1 / 2}\left(\operatorname{Re}\left(\left\|B^{(i-1)}\right\|^{-2}\right) X_{i}+\operatorname{Im}\left(\left\|B^{(i-1)}\right\|^{-2}\right) X_{i+1}\right)$ if $i=$ $s+1, \cdots, m$ with $i-(s+1)$ even, and $u_{B, i}=\left(\left\|X_{i-1}\right\|^{2}+\left\|X_{i}\right\|^{2}\right)^{1 / 2}$ $\left(-\operatorname{Im}\left(\left\|B^{(i-1)}\right\|^{-2}\right) X_{i}+\operatorname{Re}\left(\left\|B^{(i-1)}\right\|^{-2}\right) X_{i+1}\right)$ if $i=s+1, \cdots, m$ with $i-$ $(s+1)$ odd. Denote by $\pi_{B, i}: \mathbb{R}^{m} \rightarrow \mathbb{R} u_{B, i}, i=1,2, \cdots, s$ the orthogonal projection mappings to the 1-dimensional eigenspaces of $Q$, resp. $\pi_{B, i}$ : $\mathbb{R}^{m} \rightarrow \mathbb{R} u_{B, i}+\mathbb{R} u_{B, i+1}, i=s+1, \cdots, m$ with $i-(s+1)$ even, the orthogonal projection mappings to the irreducible 2-dimensional eigenspaces of $Q$. Then, for all $k \geq 0, a_{0}, \cdots, a_{k} \in \mathbb{Z}$, we have $\pi_{B, i}\left(\sum_{j=0}^{k} a_{j} Z_{j}\right)=$ $\left(\sum_{j=0}^{k} a_{j} \beta^{(i-1)^{j}}\right)\left\|X_{i}\right\|^{-1} u_{B, i}, i=1,2, \cdots, s$ and, for all $i=s+1, \cdots, m$ with $i-(s+1)$ even, $\pi_{B, i}\left(\sum_{j=0}^{k} a_{j} Z_{j}\right)=$

$$
\frac{\left(\begin{array}{cc}
\operatorname{Re}\left(\sum_{j=0}^{k} a_{j} \beta^{(i-1)^{j}}\right) & \operatorname{Im}\left(\sum_{j=0}^{k} a_{j} \beta^{(i-1)^{j}}\right) \\
-\operatorname{Im}\left(\sum_{j=0}^{k} a_{j} \beta^{(i-1)^{j}}\right) & \operatorname{Re}\left(\sum_{j=0}^{k} a_{j} \beta^{(i-1)^{j}}\right)
\end{array}\right)\left(\begin{array}{c}
u_{B, i} \\
u_{B, i+1}
\end{array}\right)}{\left(\sum_{k=0}^{m-1}\left|\beta^{(i-1)}\right|^{2 k}\right)^{1 / 2}} .
$$

Proof. It suffices to apply the real and complex embeddings of $\mathbb{Q}(\beta)$ to the relation

$$
\pi_{B}\left(\sum_{j=0}^{k} a_{j} Z_{j}\right)=\left(\left(\sum_{j=0}^{k} a_{j} Z_{j}\right) \cdot B\right)\|B\|^{-2} B=\left(\sum_{j=0}^{k} a_{j} \beta^{j}\right)\|B\|^{-2} B:
$$

for complex embeddings, $\left\|X_{i}\right\|^{2}+\left\|X_{i+1}\right\|^{2}=\sum_{k=0}^{m-1}\left|\beta^{(i-1)}\right|^{2 k}$ and $\left\|B^{(i-1)}\right\|^{-2} B^{(i-1)}$ means:

$$
\begin{gathered}
\left(\begin{array}{cc}
\operatorname{Re}\left(\left\|B^{(i-1)}\right\|^{-2}\right) & \operatorname{Im}\left(\left\|B^{(i-1)}\right\|^{-2}\right) \\
-\operatorname{Im}\left(\left\|B^{(i-1)}\right\|^{-2}\right) & \operatorname{Re}\left(\left\|B^{(i-1)}\right\|^{-2}\right)
\end{array}\right)\left(\begin{array}{c}
X_{i} \\
X_{i+1}
\end{array}\right)= \\
\frac{1}{\left(\left\|X_{i}\right\|^{2}+\left\|X_{i+1}\right\|^{2}\right)^{1 / 2}}\left(\begin{array}{c}
u_{B, i} \\
u_{B, i+1}
\end{array}\right) .
\end{gathered}
$$

The explicit expressions given above will allow us below to compare the "geometric" Rauzy fractals deduced from the present study and the "algebraic" Rauzy fractal. Before stating the main theorem about the existence of canonical cut-and-projection schemes associated with the betaintegers when $\beta$ is a general (non-integer) Perron number, let us first consider the case of equality $u=u_{B}$ and show that it is rarely occuring.

Proposition 3.3. The equality $u=u_{B}$ holds if and only if $\beta$ is a Pisot number, root $>1$ of the polynomial $X^{2}-a X-1$, with $a \geq 1$. 
Proof. The condition $u=u_{B}$ is equivalent to $V_{1}$ colinear to $B$, that is $\xi_{j, 1} \beta^{-j+1}=$ a non-zero constant, for all $j=1,2, \cdots, m$. The condition is sufficient: if $\beta$ is such a Pisot number, such equalities hold. Conversely, if such equalities hold, this implies in particular that $\xi_{1,1} \beta^{-1+1}=\xi_{m, 1} \beta^{-m+1}$. Thus we obtain $a_{0} \beta^{m-2}=1$, that is necessarily $m=2$ and $a_{0}=$ 1 . The Perron number $\beta$ is then a Pisot number of negative conjugate $-\beta^{-1}$ which satisfies $\beta^{2}-a_{1} \beta-1=0$, where $a_{1}=\beta-\beta^{-1}$ is an integer greater than or equal to 1 . This is the only possibility of quadratic Pisot number of norm -1 ([Fro1], Lemma 3).

Theorem 3.5. Denote by $E$ the line $\mathbb{R} u_{B}$ in $\mathbb{R}^{m}$. There exist two canonical cut-and-project schemes $E \stackrel{p_{1}}{\longleftarrow}\left(E \times D \simeq \mathbb{R}^{m}, \mathbb{Z}^{m}\right) \stackrel{p_{2}}{\longrightarrow} D$ associated with $\mathbb{Z}_{\beta} \subset E$ (see the definitions in the Appendix). They are given by, in case (i): the orthogonal projection mapping $\pi_{B}$ as $p_{1}, \oplus_{F} F$ as internal space $D, p_{2}=\oplus_{F} \pi_{F}$, where the sums are over all irreducible ${ }^{t} Q$-invariant subspaces $F$ of $\mathbb{R}^{m}$ except $\mathbb{R} u$ and where $\pi_{F}$ is the projection mapping to $F$ along its ${ }^{t} Q$-invariant complementary space, in case (ii): as $p_{1}$ the orthogonal projection mapping $\pi_{B}, \oplus_{F} F$ as internal space $D$ where the sum is over all irreducible $Q$-invariant subspaces $F$ of $\mathbb{R}^{m}$ except $E$, as $p_{2}$ the sum $\oplus_{i \neq 1} \pi_{B, i}$ of all the orthogonal projection mappings except $\pi_{B, 1}=\pi_{B}$; in the case (ii), the internal space $D$ is orthogonal to the line $\mathbb{R} u$.

Proof. In both cases, the fact that $p_{2}\left(\mathbb{Z}^{m}\right)$ is dense in $D$ arises from Kronecker's theorem (Appendix B in [Mey]): since $\beta$ is an algebraic integer of degree $m$, the $m$ real numbers $1=\beta^{0}, \beta^{1}, \cdots, \beta^{m-1}$ are linearly independent over $\mathbb{Q}$. Hence, for all $\epsilon>0$ and all $m$-tuple of real numbers $x_{0}, x_{1}, \cdots, x_{m-1}$ such that the vector (say) $x={ }^{t}\left(x_{0} x_{1} \ldots x_{m-1}\right)$ belongs to $D$, there exist a real number $w$ and $m$ rational integers $u_{0}, u_{1}, \cdots, u_{m-1}$ such that $\left|x_{j}-\beta^{j} w-u_{j}\right| \leq \epsilon / \sqrt{m}$ for all $j=0,1, \ldots, m-$ 1. In other terms, there exists a point $u={ }^{t}\left(u_{0}, u_{1}, \cdots, u_{m-1}\right) \in \mathbb{Z}^{m}$ such that its image $p_{1}(u)$ is $w B \in \mathbb{R} u_{B}$ and its image $p_{2}(u)$ is close to $x$ up to $\epsilon$. Hence the result. As for the restriction of the projection mapping $p_{1}=\pi_{B}=\pi_{B, 1}: \mathbb{R}^{m} \rightarrow E$ to the lattice $\mathbb{Z}^{m}$, it is injective after Theorem 3.4. The orthogonality between $D$ and $u$ comes directly from Corollary 3.3 .

The mapping $p_{1}\left(\mathbb{Z}^{m}\right) \rightarrow D: x \rightarrow x^{*}=p_{2} \circ\left(p_{1_{\mathbb{Z}^{m}}}\right)^{-1}(x)$ will be denoted by the same symbol $(.)^{*}$ in the cases (i) and (ii), the context making the difference.

Proposition 3.4. Let $\beta$ be a Pisot number, root $>1$ of the polynomial $X^{2}-a X-1$, with $a \geq 1$. Put $c_{a}=\frac{(1+a \beta)\lfloor\beta\rfloor}{\sqrt{2+a \beta(\beta-1)}}$. Then the two canonical cut-and-project schemes given by (i) and (ii) in Theorem 3.5 are 
identical and the inclusion of $\mathbb{Z}_{\beta}\|B\|^{-1} u_{B}$ in the following model set holds: $\mathbb{Z}_{\beta}\|B\|^{-1} u_{B}=\pi_{B}(\mathcal{L} \cup(-\mathcal{L})) \subset\left\{v \in \pi_{B}\left(\mathbb{Z}^{2}\right) \mid v^{*} \in\left[-c_{a} u_{B, 2},+c_{a} u_{B, 2}\right]\right\}$ where $u_{B, 2}={ }^{t}(-\beta 1)\|B\|^{-1}$.

Proof. The two cut-and-project schemes are identical: by Proposition 3.3 the equality $u=u_{B}$ holds and the line $\mathbb{R} u_{B, 2}$, which is obviously orthogonal to the line $\mathbb{R} u_{B}$, is ${ }^{t} Q$-invariant. Now, if $g$ denotes an arbitrary element of $\mathcal{L}$, it can be written $g=x_{k}\left({ }^{t} Q\right)^{k} Z_{0}+x_{k-1}\left({ }^{t} Q\right)^{k-1} Z_{0}+$ $\cdots+x_{1}\left({ }^{t} Q\right) Z_{0}+x_{0} Z_{0}$ for a certain integer $k \geq 0$ with $x_{i} \in A$ and $\left(x_{j}, x_{j-1}, \cdots, x_{1}, x_{0}, 0,0, \cdots\right)<\left(c_{1}, c_{2}, \cdots\right)$ for all $j, 0 \leq j \leq k$. We have $Z_{0}=s u+s^{\perp} u_{B, 2}$ with $s=\|B\|^{-1}$ and $s^{\perp}=-\beta\|B\|^{-1}$. Then $g=\sum_{j=0}^{k} x_{j}\left({ }^{t} Q\right)^{j} Z_{0}=\sum_{j=0}^{k} x_{j}\left(s \beta^{\jmath} u+s^{\perp}(-1)^{j} \beta^{-j} u_{B, 2}\right)$. Thus $p_{2}(g)=$ $s^{\perp} \sum_{j=0}^{k} x_{j}(-1)^{j} \beta^{-j} u_{B, 2}$ and $\left\|p_{1}(g)^{*}\right\|=\left\|p_{2}(g)\right\| \leq\left|s^{\perp}\right|\lfloor\beta\rfloor \sum_{j=0}^{+\infty} \beta^{-j}$ $=\left|s^{\perp}\right|\lfloor\beta\rfloor \frac{1}{1-\beta^{-1}}$ which is equal to $c_{a}$ since $\|B\|=\sqrt{2+a \beta}$. This constant is independent of $k$. Hence we have $p_{1}(g) \subset\left\{v \in \pi_{B}\left(\mathbb{Z}^{2}\right) \mid v^{*} \in\right.$ $\left.\left[-c_{a} u_{B, 2},+c_{a} u_{B, 2}\right]\right\}$ and the claim.

Let $\mathcal{C}=\left\{\sum_{j=0}^{m-1} \alpha_{j} Z_{j} \mid \alpha_{j} \in[0 ; 1]\right.$ for all $\left.j=0,1, \cdots, m-1\right\}$ be the $m$-cube at the origin. For all irreducible ${ }^{t} Q$ - invariant subspace $F$ of $\mathbb{R}^{m}$, put $\delta_{F}=\max _{x \in \mathcal{C}}\left\|\pi_{F}(x)\right\|, \lambda_{F}$ the absolute value of the eigenvalue of ${ }^{t} Q$ on $F$ and $c_{F}=\lfloor\beta\rfloor \frac{\delta_{F}}{1-\lambda_{F}^{m}}$. Denote by $\Omega_{F}$ the closed interval centred at 0 in $F$ of length $2 c_{F}$ if $\operatorname{dim} F=1$, resp. the closed disc centred at 0 in $F$ of radius $c_{F}$ if $\operatorname{dim} F=2$.

Theorem 3.6. Let $\beta$ be a Pisot number of degree $m \geq 2$ and $\Omega=\oplus_{F} \Omega_{F}$ where the sum is over all irreducible ${ }^{t} Q$ - invariant subspace $F$ of $\mathbb{R}^{m}$ except $\mathbb{R} u$. Then the inclusion of $\mathbb{Z}_{\beta}\|B\|^{-1} u_{B}$ in the following model set defined by $\Omega$ holds: $\mathbb{Z}_{\beta}\|B\|^{-1} u_{B}=p_{1}(\mathcal{L} \cup(-\mathcal{L})) \subset\left\{v \in p_{1}\left(\mathbb{Z}^{m}\right) \mid v^{*} \in\right.$ $\Omega\}$ in the cut-and-project scheme given by the case (i) in Theorem 3.5.

Proof. If $g=\sum_{j=0}^{k} x_{j} Z_{j} \in \mathcal{L}$ with $k=d m-1$, and $d \geq 1$ an integer, then

$$
g=\sum_{q=0}^{d-1} \sum_{l=0}^{m-1} x_{q m+l}\left({ }^{t} Q\right)^{q m} Z_{l}=\sum_{q=0}^{d-1}\left({ }^{t} Q\right)^{q m}\left(\sum_{l=0}^{m-1} x_{q m+l} Z_{l}\right) .
$$

Hence $p_{1}(g)^{*}=p_{2}(g)=\oplus_{F} \pi_{F}(g)=\sum_{F} \sum_{q=0}^{d-1}\left[\left({ }^{t} Q_{\mid F}\right)^{q m} \pi_{F}\left(\sum_{l=0}^{m-1} x_{q m+l} Z_{l}\right)\right]$ with:

$\left\|\pi_{F}(g)\right\| \leq \sum_{q=0}^{d-1}\lfloor\beta\rfloor \lambda_{F}^{q m}\left\|\pi_{F}\left(\sum_{l=0}^{m-1} Z_{l}\right)\right\| \leq\lfloor\beta\rfloor \delta_{F} \sum_{q=0}^{+\infty} \lambda_{F}^{q m}=\lfloor\beta\rfloor \frac{\delta_{F}}{1-\lambda_{F}^{m}}=c_{F}$. 
This constant is independent of $d$, hence of $k=d m-1$. It is easy to check that it is an upper bound for $\left\|p_{2}(g)\right\|$ if $k \not \equiv-1(\bmod m)$ and also for all $g \in-\mathcal{L}$. We deduce the claim.

Corollary 3.7. If $\beta$ is a Pisot number of degree $m \geq 2$, then $\mathbb{Z}_{\beta}$ is a Meyer set.

Proof. If $\beta$ is a Pisot number, the set $\mathbb{Z}_{\beta}$, viewed as the set of vertices of an aperiodic tiling, is obtained by concatenation of prototiles on the line, which are in finite number by Thurston [Th]. And it is relatively dense by construction. Now, by Theorem 3.6 it is included in a model set. This proves the claim (see the Appendix).

In both cases of cut-and-project scheme, as given by Theorem 3.5 where the duality between the matrices $Q$ and ${ }^{t} Q$ clearly appears, the internal space represents the contracting hyperplane, whereas the line $\mathbb{R} u$ is the expanding direction, when $\beta$ is a Pisot number. The duality between both cut-and-project schemes is connected to the substitutive approach by the following (Arnoux and Ito [AI], Chap. 7 in Pytheas Fogg [PF]): the abelianized $Z_{k}^{\prime}$ of the iterates of the substitution satisfy $Z_{k+1}^{\prime}=Q Z_{k}^{\prime}$, and gather now about the line $\mathbb{R} B$. If one takes the projection on $\mathbb{R} B$ of the new set $\mathcal{L}^{\prime}$ (defined similarly as $\mathcal{L}$ ) along the other eigenspaces, one recovers $\mathbb{Z}_{\beta}$ (up to a scalar factor). A striking feature of the internal spaces is that the numeration in base $\beta^{(j)}$ (conjugates of $\beta$ ) appears as canonical ingredient to control the distance between a point of $\mathcal{L}$ and its orthogonal projection to the expanding line $\mathbb{R} u$, in particular at infinity.

Definition 3.1. Let $\beta$ be a Pisot number of degree $m \geq 2$. The closure $\overline{\left(\mathbb{Z}_{\beta}^{+}\|B\|^{-1} u_{B}\right)^{*}}$ of the set $p_{2}(\mathcal{L})$ is called the canonical acceptance window associated with the set of beta-integers $\mathbb{Z}_{\beta}^{+}$in both cases (case (i) or (ii) in Theorem 3.5) of cut-and-project scheme: in the case (i) it will be denoted by $\mathcal{R}_{i}$ and in the case (ii) by $\mathcal{R}$.

The notations $\mathcal{R}$ and $\mathcal{R}_{i}\left(\mathcal{R}_{i} \subset \Omega\right.$ by Theorem 3.6) with an " $\mathcal{R} "$ like Rauzy are used to recall the close similarity between these sets and the Rauzy fractal (Rauzy [Ra], Arnoux and Ito [AI], Messaoudi [Me], Ito and Sano [IS], Chap. 7 in Pytheas Fogg [PF]). The fact is that the set $\mathcal{R}$ is exactly the Rauzy fractal up to the multiplication by a non-zero scalar factor on each irreducible $Q$-invariant subspace (by definition we will speak of quasi-dilation). Let us show it on an example.

"Tribonacci" case [Me]: let us consider the irreducible polynomial $P(X)=X^{3}-X^{2}-X-1$. Its dominant root is denoted by $\beta$, and $\alpha$ and $\bar{\alpha}$ are the two other complex conjugates roots of $P(X)$. In this case, the Rauzy fractal is "algebraically" defined by $\mathcal{E}:=\left\{\sum_{i=3}^{\infty} \epsilon_{i} \alpha^{i} \mid \epsilon_{i} \in\right.$ 
$\{0,1\}$ and $\epsilon_{i} \epsilon_{i+1} \epsilon_{i+2}=0$ for all integer $\left.i \geq 3\right\}$. The condition imposed on the sequence $\left(\epsilon_{i}\right)_{i \geq 3}$ is exactly that given by the conditions of Parry. Indeed ([Fro1] and section 2), $d_{\beta}(1)=0.111$ and the lexicographical maximal sequence is $c_{1} c_{2} c_{3} \cdots=(110)^{\omega}$. Now (Proposition 3.2) $B^{(1)}=$ ${ }^{t}\left(1 \alpha \alpha^{2}\right)$ and $\left\|X_{2}\right\|^{2}+\left\|X_{3}\right\|^{2}=1+\alpha \bar{\alpha}+\alpha^{2} \bar{\alpha}^{2}=\beta$. We deduce that $\mathcal{R}=\beta^{-1 / 2} \mathcal{E}=\pi_{B, 2}(\mathcal{L})$ with the following (metric) identification of $\mathbb{C}$ : $\phi(\mathbb{C})=\mathbb{R} u_{B, 2}+\mathbb{R} u_{B, 3}$ where $\phi$ is the isometry which sends the vector $\left(\begin{array}{l}1 \\ 0\end{array}\right)$, resp. $\left(\begin{array}{l}0 \\ 1\end{array}\right)$, to $\left(\begin{array}{l}u_{B, 2} \\ u_{B, 3}\end{array}\right)$, resp. to $\left(\begin{array}{c}u_{B, 3} \\ -u_{B, 2}\end{array}\right)$.

Proposition 3.5. The canonical acceptance window $\mathcal{R}$ (relative to the case (ii) of cut-and-project scheme in Theorem 3.5) is compact and connected. Its interior int $(\mathcal{R})$ is simply connected, contains the origin. The set $\mathcal{R}$ is such that: (i) $\overline{\text { int }(\mathcal{R})}=\mathcal{R}$; (ii) it induces a tiling of the internal space $D$ modulo the lattice $\phi(\mathbb{Z}+\mathbb{Z} \alpha): \quad D=\bigcup_{z \in \phi(\mathbb{Z}+\mathbb{Z} \alpha)}(\mathcal{R}+z)$; (iii) $(\mathcal{R}+z) \cap \operatorname{int}\left(\mathcal{R}+z^{\prime}\right)=\emptyset$ for all $z, z^{\prime} \in \phi(\mathbb{Z}+\mathbb{Z} \alpha), z \neq z^{\prime}$.

Proof. Since $\mathcal{R}=\beta^{-1 / 2} \mathcal{E}$, we deduce the properties of $\mathcal{R}$ from those of $\mathcal{E}$ already established in Rauzy [Ra], Messaoudi [Me] and [Me1].

Proposition 3.6. The boundary of $\mathcal{R}$ is a fractal Jordan curve. A point $z$ belongs to the boundary of $\mathcal{R}$ if and only if it admits at least 2 distinct Rényi $\alpha$-expansions. A point belonging to the boundary of $\mathcal{R}$ admits 2 or 3 distinct Rényi $\alpha$-expansions, never more.

Proof. The properties of the boundary of $\mathcal{E}$ are given in Ito and Kimura [IK] and Messaoudi [Me1]). Hence the claim.

The properties of $\mathcal{R}_{i}$ follow from the equality: $p_{2}(\mathcal{R})=\mathcal{R}_{i}$, where $p_{2}$ refers to the case (i) of cut-and-project scheme in Theorem 3.5, and from Proposition 3.5 and 3.6: in particular, it has also a fractal boundary. We will speak of "geometrical" Rauzy fractals for $\mathcal{R}$ and $\mathcal{R}_{i}$ and of "algebraic" Rauzy fractal for $\mathcal{E}$. They are similar objects as far as they concentrate all the information about the beta-integers and the completions of their real and complex embeddings (Rauzy $[\mathrm{Ra}]$ ). The respective canonical acceptance windows associated with $\mathbb{Z}_{\beta}$ are $\mathcal{R} \cup(-\mathcal{R})$ and $\mathcal{R}_{i} \cup\left(-\mathcal{R}_{i}\right)$ in the two cut-and-project schemes.

\section{Additive properties of $\mathbb{Z}_{\beta}$}

In this section, $\beta$ will be a Pisot number of degree $m \geq 2$.

A) Cones, generators and semi-groups. - We will show that any element of $\mathcal{L}$ is generated by a finite number of elements of $\mathcal{L}$ of small norm, over $\mathbb{N}$. By projection to $E$ by $\pi_{B}$, the ambiant 1-dimensional space of the beta-integers (Theorem 3.5), this will imply the same property 
for $\mathbb{Z}_{\beta}$. This finiteness property, stated in Corollary 4.5 , constitutes a refinement of Theorem 4.12 (i) (Lagarias) for the Meyer sets $\mathbb{Z}_{\beta}$.

First let us fix the notations and simplify them somehow. Let $\pi: \mathbb{R}^{m} \rightarrow$ $\mathbb{R} u$ be the projection mapping along its ${ }^{t} Q$-invariant complementary space (instead of denoting it by $\pi_{\mathbb{R} u}$ ), and $p_{2}$ the projection mapping of the cutand-project scheme (i) in Theorem 3.5. Let $\pi^{\|}: \mathbb{R}^{m} \rightarrow \mathbb{R} u$ be the orthogonal projection mapping and $\pi^{\perp}=I d-\pi^{\|}\left(\pi^{\perp}\right.$ is the mapping $\oplus_{i \neq 1} \pi_{b, i}$ in the case (ii) of cut-and-project scheme in Theorem 3.5). The basic ingredient will be the construction of semi-groups of finite type associated with cones whose axis of revolution is the expanding line $\mathbb{R} u$, following an idea of Lind [Li] in another context. Truncating them in a suitable way at a certain distance of the origin will be the key for finding generators of $\mathcal{L}$ over $\mathbb{N}$. In the first Lemma we will consider the possible angular openings of these cones around the expanding line $\mathbb{R} u$ for catching the points of $\mathcal{L}$. For $\theta>0$, define the cone $K_{\theta}:=\left\{x \in \mathbb{R}^{m} \mid \theta\left\|p_{2}(x)\right\| \leq\|\pi(x)\|, 0 \leq \pi(x) \cdot u\right\}$. For $r, w>0$, define $K_{\theta}(r):=\left\{x \in K_{\theta} \mid\|\pi(x)\| \leq r\right\}, \quad K_{\theta}(r, w):=$ $\left\{x \in K_{\theta} \mid r \leq\|\pi(x)\| \leq w\right\}$. If $\mathcal{A}$ is an arbitrary subset of $\mathbb{R}^{m}$, denote by $s g(\mathcal{A}):=\left\{\sum_{\text {finite }} m_{i} x_{i} \mid m_{i} \in \mathbb{N}, x_{i} \in \mathcal{A}\right\}$ the semigroup generated by $\mathcal{A}$. Let $\rho$ be the covering radius of the subset $\mathcal{L} \cup(-\mathcal{L})$ with respect to the band $\mathcal{R}_{i} \times \mathbb{R} u: \quad \rho$ is the smallest positive real number such that for any $z \in \mathbb{R}^{m}$ such that $p_{2}(z) \in \mathcal{R}_{i}$ the closed ball $B(z, \rho)$ contains at least one element of $\mathcal{L} \cup(-\mathcal{L})$. A lower bound of $\rho$ is given by the covering radius $\sqrt{m} / 2$ of the lattice $\mathbb{Z}^{m}$. Referring $\mathbb{R}^{m}$ to the basis $\left\{B, V_{2}, V_{3}, \cdots, V_{s+2 t}\right\}$ and using Corollary 3.3 and Theorem 3.6 we easily deduce the following upper bound of $\rho: \frac{1}{2}\|B\|^{-1}+\sum_{F} c_{F}$, where the sum $\sum_{F}$ means there and in the following everywhere it will be used "the sum over all irreducible ${ }^{t} Q$-invariant subspaces $F$ of $\mathbb{R}^{m}$ except $\mathbb{R} u$ ". The notation $\operatorname{diam}(\cdot)$ will be put for the diameter of the set $(\cdot)$ in the following.

Proposition 4.1. (i) For all $\theta>0$, there exists an integer $j_{0}=j_{0}(\theta) \geq$ 0 such that $Z_{j} \in K_{\theta}$ for all $j \geq j_{0}$; (ii) if ${ }^{t} Q$ is nonnegative, and $\min \left\{\xi_{j, 1} \mid j=1,2, \cdots, m\right\}>2\left\|V_{1}\right\|\left(\operatorname{diam}\left(\mathcal{R}_{i}\right)\right)$, then the following equality $j_{0}(\theta)=0$ holds for all $0<\theta<\theta_{\text {min }}$, where $\theta_{\text {min }}:=-2+$ $\left(\operatorname{diam}\left(\mathcal{R}_{i}\right)\right)^{-1}\left\|V_{1}\right\|^{-1} \min \left\{\xi_{j, 1} \mid j=1,2, \cdots, m\right\}$.

Proof. (i) Let $\theta>0$. We have just to prove that $\pi\left(Z_{j}\right) \cdot u$ tends to $+\infty$ and not to $-\infty$ when $j$ goes to $+\infty$. Let $j \geq 0$. Write $Z_{j}=\pi\left(Z_{j}\right)+p_{2}\left(Z_{j}\right)=\pi^{\|}\left(Z_{j}\right)+\pi^{\perp}\left(Z_{j}\right)$; hence $\left\|\pi\left(Z_{j}\right)-\pi^{\|}\left(Z_{j}\right)\right\|=$ $\left\|\pi^{\perp}\left(Z_{j}\right)-p_{2}\left(Z_{j}\right)\right\| \leq\left\|\pi^{\perp}\left(Z_{j}\right)\right\|+\left\|p_{2}\left(Z_{j}\right)\right\|=\left\|\pi^{\perp}\left(Z_{j}-\pi\left(Z_{j}\right)\right)\right\|+$ $\left\|p_{2}\left(Z_{j}\right)\right\| \leq 2\left\|p_{2}\left(Z_{j}\right)\right\| \leq 2 \operatorname{diam}\left(\mathcal{R}_{i}\right)$. On the other hand $\| \pi_{B}\left(\pi^{\|}\left(Z_{j}\right)\right)-$ $\pi_{B}\left(Z_{j}\right)\|=\|\left(Z_{j} \cdot u\right)\left(u_{B} \cdot u\right) u_{B}-\|B\|^{-1} \beta^{j} u_{B} \|=\mid\left(Z_{j} \cdot u\right)\left(u_{B} \cdot u\right)-$ $\|B\|^{-1} \beta^{j} \mid=\left\|\pi_{B}\left(\pi^{\perp}\left(Z_{j}\right)\right)\right\| \leq\left\|\pi^{\perp}\left(Z_{j}\right)\right\| \leq \operatorname{diam}(\mathcal{R})$. Hence, since 
$u_{B} \cdot u>0$ (Theorem 3.2), $\left|Z_{j} \cdot u-\left(u_{B} \cdot u\right)^{-1}\|B\|^{-1} \beta^{j}\right| \leq\left(u_{B} \cdot u\right)^{-1} \operatorname{diam}(\mathcal{R})$. Consequently $\left\|\pi\left(Z_{j}\right)-\left(u_{B} \cdot u\right)^{-1}\right\| B\left\|^{-1} \beta^{j} u\right\|=\| \pi\left(Z_{j}\right)-\pi^{\|}\left(Z_{j}\right)+\pi^{\|}\left(Z_{j}\right)-$ $\left(u_{B} \cdot u\right)^{-1}\|B\|^{-1} \beta^{j} u\|\leq\| \pi\left(Z_{j}\right)-\pi^{\|}\left(Z_{j}\right)\|+\| \pi^{\|}\left(Z_{j}\right)-\left(u_{B} \cdot u\right)^{-1}\|B\|^{-1} \beta^{j} u \|$ $\left.\leq 2 \operatorname{diam}\left(\mathcal{R}_{i}\right)+\left(u_{B} \cdot u\right)^{-1}\right) \operatorname{diam}(\mathcal{R})$. The quantity $\pi\left(Z_{j}\right) \cdot u$ tends to $+\infty$ as $\left(u_{B} \cdot u\right)^{-1}\|B\|^{-1} \beta^{j}$ when $j \rightarrow+\infty$. Then there exists $j_{0}$ such that $Z_{j} \cdot u \geq 2 \operatorname{diam}\left(\mathcal{R}_{i}\right)+\left(\left(u_{B} \cdot u\right)^{-1}+\theta\right) \operatorname{diam}\left(\mathcal{R}_{i}\right)$, for all $j \geq j_{0}$. As a consequence $\pi\left(Z_{j}\right) \cdot u \geq Z_{j} \cdot u-2 \operatorname{diam}\left(\mathcal{R}_{i}\right) \geq\left(\left(u_{B} \cdot u\right)^{-1}+\theta\right) \operatorname{diam}\left(\mathcal{R}_{i}\right)>$ 0 for all $j \geq j_{0}$. We claim that $Z_{j} \in K_{\theta}$ for all $j \geq j_{0}$. Indeed, since $\left\|p_{2}\left(Z_{j}\right)\right\| \leq \operatorname{diam}\left(\mathcal{R}_{i}\right)$, the inequalities hold: $\theta\left\|p_{2}\left(Z_{j}\right)\right\| \leq(\theta+$ $\left.\left(u_{B} \cdot u\right)^{-1}\right)\left\|p_{2}\left(Z_{j}\right)\right\| \leq\left(\theta+\left(u_{B} \cdot u\right)^{-1}\right) \operatorname{diam}\left(\mathcal{R}_{i}\right) \leq \pi\left(Z_{j}\right) \cdot u=\left\|\pi\left(Z_{j}\right)\right\|$ for all $j \geq j_{0}$.

(ii) If ${ }^{t} Q$ is nonnegative the coefficients $a_{i}$ in $P(X)$ are nonnegative with $a_{0} \neq 0$ and at least one of the coefficients $a_{k}, k \geq 1$, is non-zero since $\beta$ is assumed to be a Pisot number and not a Salem number. Hence (Proposition 3.1), since $P^{\prime}(\beta)>0$, we have $\left\|V_{1}\right\|^{-1} \xi_{1,1}=\left\|\pi^{\|}\left(Z_{0}\right)\right\|=$ $Z_{0} \cdot u=\frac{a_{0}}{\beta P^{\prime}(\beta)}>0$ and $\left\|V_{1}\right\|^{-1} \xi_{j+1,1}=\left\|\pi^{\|}\left(Z_{j}\right)\right\|=Z_{j} \cdot u$, for all $j=$ $1,2, \cdots m-1$ with $\left\|V_{1}\right\|^{-1} \min \left\{\xi_{j, 1} \mid j=1,2, \cdots m-1\right\} \geq \frac{a_{0}}{\beta^{m} P^{\prime}(\beta)}>0$. Because $\left\{Z_{0}, Z_{1}, \cdots, Z_{m-1}\right\}$ is the canonical basis of $\mathbb{R}^{m}$, any $Z_{j}, j \geq m$, can be written as a combination of the elements of this basis with positive coefficients. Hence, $Z_{j} \cdot u \geq\left\|V_{1}\right\|^{-1} \min \left\{\xi_{l, 1} \mid l=1,2, \cdots, m-1\right\}$ for all $j \geq 0$. But the relation $Z_{j}=\pi\left(Z_{j}\right)+p_{2}\left(Z_{j}\right)=\pi^{\|}\left(Z_{j}\right)+\pi^{\perp}\left(Z_{j}\right)$ implies that $\pi\left(Z_{j}\right)-\pi^{\|}\left(Z_{j}\right)=\pi^{\perp}\left(Z_{j}\right)-p_{2}\left(Z_{j}\right)$. Hence, $\left|\pi\left(Z_{j}\right) \cdot u-Z_{j} \cdot u\right| \leq$ $\left\|\pi^{\perp}\left(Z_{j}\right)\right\|+\left\|p_{2}\left(Z_{j}\right)\right\| \leq 2\left\|p_{2}\left(Z_{j}\right)\right\| \leq 2\left(\operatorname{diam}\left(\mathcal{R}_{i}\right)\right)$ for all $j \geq 0$. Therefore $\pi\left(Z_{j}\right) \cdot u \geq\left\|V_{1}\right\|^{-1} \min \left\{\xi_{l, 1} \mid l=1,2, \cdots, m-1\right\}-2 \operatorname{diam}\left(\mathcal{R}_{i}\right)$ which is $>0$ by assumption for all $j \geq 0$. Hence, by definition of $\theta_{\text {min }}$, $\pi\left(Z_{j}\right) \cdot u=\left\|\pi\left(Z_{j}\right)\right\| \geq \theta_{\min }\left(\operatorname{diam}\left(\mathcal{R}_{i}\right)\right) \geq \theta_{\min }\left\|p_{2}\left(Z_{j}\right)\right\| \geq \theta\left\|p_{2}\left(Z_{j}\right)\right\|$ for all $j \geq 0$ and $0<\theta \leq \theta_{\text {min }}$. We deduce that $Z_{j} \in K_{\theta}$ for all $j \geq 0$ and $0<\theta \leq \theta_{\min }$. Let us observe that the conditions of the present assertion are generally not fulfilled.

We now turn to the question of generating the elements of $\mathcal{L}$ by a finite number of them over $\mathbb{N}$. The idea we will follow is simple: let us consider the set of the semi-groups generated by a finite number of (arbitrary) elements of $\mathcal{L} \cap K_{\theta}$ for all $\theta>0$; in this set, we will show the existence of semi-groups $\left(\theta>0\right.$ fixed) containing $K_{2 \theta} \cap \mathcal{L}$, that is containing $\mathcal{L}$ except a finite number of elements of $\mathcal{L}$ close to the origin. Then we will minimize this finite number of excluded elements. For this we will consider the maximal possible values of $\theta$. In final this will provide a suitable value of $\theta$ and a control of the norms of the generating elements of the semi-group which will contain $\mathcal{L}$. 
Lemma 4.1. (Lind [Li]) Let $\theta>0$. If $\delta=(2 \theta+2)^{-1}$ and $x \in K_{2 \theta}$ with $\|\pi(x)\|=\pi(x) \cdot u>4$, then $\left[x-K_{\theta}(1,3)\right] \cap K_{2 \theta}$ contains a ball of radius $\delta$.

Proof. [Li] Take $y=2 u+3(\pi(x) \cdot u)^{-1} p_{2}(x)$. We will show that the ball centred at $x-y$ and of radius $\delta$ satisfies our claim. Suppose $\|z\|<\delta$. Then $x-y+z \in K_{2 \theta}$. Indeed,

$$
\begin{gathered}
2 \theta\left\|p_{2}(x-y+z)\right\| \leq 2 \theta\left[\left(1-3(\pi(x) \cdot u)^{-1}\right)\left\|p_{2}(x)\right\|+\delta\right] \\
\leq\left[1-3(\pi(x) \cdot u)^{-1}\right](\pi(x) \cdot u)+2 \theta(2 \theta+2)^{-1}=(\pi(x) \cdot u)-2-2(2 \theta+2)^{-1}
\end{gathered}
$$
but $p_{2}(y)=2$. We deduce $2 \theta\left\|p_{2}(x-y+z)\right\| \leq \pi(x-y+z) \cdot u$. Let us show that $y-z \in K_{\theta}$. We have $2 \theta\left\|p_{2}(y)\right\|=6 \theta(\pi(x) \cdot u)^{-1}\left\|p_{2}(x)\right\| \leq$ $3(\pi(x) \cdot u)^{-1}(\pi(x) \cdot u)=3$. Therefore $\theta\left\|p_{2}(y-z)\right\| \leq \theta\left(\left\|p_{2}(y)\right\|+\delta\right) \leq$ $\frac{3}{2}+\theta(2 \theta+2)^{-1}=2-(2 \theta+2)^{-1} \leq \pi(y-z) \cdot u$. Now, since $\delta<1$, we have the inequalities $1 \leq \pi(y-z) \cdot u \leq 3$, establishing the result.

Theorem 4.2. Let $\theta>0$. If $r$ is such that $r>\rho(2 \theta+2)$, then $K_{2 \theta} \cap \mathcal{L} \subset s g\left(K_{\theta}(r) \cap \mathcal{L}\right)$.

Proof. Lemma 4.1 implies the following assertion: if $x \in K_{2 \theta}$ is such that $\pi(x) \cdot u>4 r$ with $r>\rho(2 \theta+2)$, then $\left[x-K_{\theta}(r, 3 r)\right] \cap K_{2 \theta}$ contains a ball of radius $r \delta>\rho$. But $\rho$ is by definition the covering radius of $\mathcal{L} \cup(-\mathcal{L})$, hence this ball intersects $\mathcal{L}$. Now, let $\mathcal{A}=K_{\theta}(4 r) \cap \mathcal{L}$ be the finite point set of $\mathcal{L}$ and let us show that $K_{2 \theta} \cap \mathcal{L} \subset s g(\mathcal{A})$. First the inclusion $K_{2 \theta}(4 r) \cap \mathcal{L} \subset \operatorname{sg}(\mathcal{A})$ holds. We now proceed inductively. Suppose $K_{2 \theta}\left(r^{\prime}\right) \cap \mathcal{L} \subset s g(\mathcal{A})$ for some $r^{\prime} \geq 4 r$. We will show that this implies $K_{2 \theta}\left(r^{\prime}+r\right) \cap \mathcal{L} \subset s g(\mathcal{A})$, which will suffice by induction. For this, let us take $g \in \mathcal{L} \cap\left[K_{2 \theta}\left(r^{\prime}+r\right) K_{2 \theta}(r)\right]$. From Lemma 4.1 and the above, there exists an element, say $y$, in $\mathcal{L}$, contained in $\left[g-K_{\theta}(r, 3 r)\right] \cap K_{2 \theta}\left(r^{\prime}\right)$. By assumption, $y \in s g(\mathcal{A})$ and $y=g-x$ for some $x \in K_{\theta}(r, 3 r) \cap \mathcal{L}_{\mathcal{L}} \subset$ $s g(\mathcal{A})$. Therefore $g=x+y \in s g(\mathcal{A})+s g(\mathcal{A}) \subset s g(\mathcal{A})$. This concludes the induction.

Lemma 4.3. For all $\theta>0$, the following set: $\mathcal{L}(\theta):=\left\{x \in \mathcal{L} \mid p_{2}(x) \in\right.$ $\left.\mathcal{R}_{i}, x \notin K_{\theta}(\rho(2 \theta+2)), x \notin K_{2 \theta}\right\}$ is finite.

Proof. The proof is clear since all $g \in \mathcal{L}$ such that $\pi(g) \cdot u>2 \rho(2 \theta+$ 2) belongs to $K_{2 \theta}$.

Define $\theta_{f}:=\max \{\theta>0 \mid \#(\mathcal{L}(\theta)$ ) is minimal $\}$ (where \#(.) denotes the number of elements of the set $(\cdot))$. If ${ }^{t} Q$ is nonnegative and the condition (ii) in Proposition 4.1 satisfied, then the equality $\#(\mathcal{L}(\theta))=0$ holds for $\theta<\theta_{\min }$ and therefore $\theta_{f} \geq \theta_{\min } / 2$.

Theorem 4.4. (Minimal decomposition). - Any element $g \in \mathcal{L} \backslash \mathcal{L}\left(\theta_{f}\right)$ can be expressed as a finite combination over $\mathbb{N}$ of elements of the finite point set $K_{\theta_{f}}\left(\rho\left(2 \theta_{f}+2\right)\right) \cap \mathcal{L}$. 
Proof. It is a consequence of Theorem 4.2 with $\theta=\theta_{f}$ and $r=\rho\left(2 \theta_{f}+\right.$ 2).

Corollary 4.5. There exist two disjoint finite subsets $\mathcal{F}=$ $\left.\left\{\|B\| \pi_{B}(g) \cdot u_{B} \mid g \in \mathcal{L}\left(\theta_{f}\right)\right)\right\} \quad$ and $\quad \mathcal{F}^{\prime}=\left\{g_{1}, g_{2}, \cdots, g_{\eta}\right\} \quad \subset$ $\left\{\|B\| \pi_{B}(g) \cdot u_{B} \mid g \in K_{\theta_{f}}\left(\rho\left(2 \theta_{f}+2\right)\right) \cap \mathcal{L}\right\}$ of $\mathbb{Z}_{\beta}^{+}$such that

$$
\mathbb{Z}_{\beta}^{+} \subset \mathcal{F} \cup \mathbb{N}\left[g_{1}, g_{2}, \cdots, g_{\eta}\right] .
$$

The generating elements $g_{i} \in \mathcal{F}^{\prime}$ satisfy: $\left\|g_{i}\right\| \leq \rho\left(2 \theta_{f}+2\right)\|B\|^{-1}\left\|V_{1}\right\|^{-1}+$ $\operatorname{diam}\left(\mathcal{R}_{i}\right)$. If the couple $\left(\mathcal{F}, \mathcal{F}^{\prime}\right)$ is such that $\eta=\# \mathcal{F}^{\prime}$ is minimal for the inclusion relation (2) and $\mathcal{F}$ is empty, then the degree $m$ of $\beta$ divides $\eta$.

Proof. To obtain the inclusion (2) it suffices to project $\mathcal{L}$ by $\pi_{B}$ and to apply Theorem 4.2 and 4.4 and Lemma 4.3. Let us show the upper bound on the norms of the elements of $\mathcal{F}^{\prime}$. If $g \in K_{\theta_{f}}\left(\rho\left(2 \theta_{f}+2\right)\right) \cap \mathcal{L}$ is decomposed as $g=\pi(g)+t$, where $t \in \mathcal{R}_{i}$, then, by Theorem 3.2 (i), we have: $\left\|\pi_{B}(g)\right\| \leq\|\pi(g)\|\|B\|^{-1}\left\|V_{1}\right\|^{-1}+\operatorname{diam}\left(\mathcal{R}_{i}\right)$. But $\|\pi(g)\| \leq$ $\rho\left(2 \theta_{f}+2\right)$. We deduce the claim. Now if $\mathbb{Z}_{\beta}^{+} \subset \mathbb{N}\left[g_{1}, g_{2}, \cdots, g_{\eta}\right]$ the group $\mathbb{Z}\left[g_{1}, g_{2}, \cdots, g_{\eta}\right]$ contains $\mathbb{Z}_{\beta}$ and the equality $\mathbb{Z}\left[\mathbb{Z}_{\beta}\right]=\mathbb{Z}\left[g_{1}, g_{2}, \cdots, g_{\eta}\right]$ necessarily holds. By Theorem 4.12 we deduce that $m$ divides $\eta$ since the rank of $\mathbb{Z}\left[\mathbb{Z}_{\beta}\right]=\mathbb{Z}\left[\mathbb{N}\left[\mathbb{Z}_{\beta}\right]\right]$ is $\eta$ when $\eta$ is the smallest integer such that the set inclusion (2) holds and that $\mathcal{F}$ is empty.

B) Preperiods in the addition of beta-integers. - The Delone set $\mathbb{Z}_{\beta}$ endowed with the usual addition and multiplication cannot have a ring structure otherwise it would contain $\mathbb{Z}$ but it is obvious that $\mathbb{Z}_{\beta}$ contains no subset of the type $\lambda \mathbb{Z}, \lambda>0$. This absence of ring structure on $\mathbb{Z}_{\beta}$ for the usual laws can be partially overcome by controlling the fractional parts of the Rényi $\beta$-expansions of $x+y$ and $x-y$ when $x, y \in \mathbb{Z}_{\beta}$. This is the aim of this paragraph to focus on the geometrical meaning of the sets $T$ and $T^{\prime}$ as stated in Theorem 4.7 and of the exponent $q$ in its Corollary 4.8 .

The projection mappings will be the ones redefined (in a simpler way) at the beginning of the subsection A). Let $R>0$ and $I$ be an interval of $\mathbb{R}$ having compact closure. Let us extend the $m$-cube $\mathcal{C}$ for reasons which will appear below. Let $\mathcal{C}^{\prime}=\left\{\sum_{j=0}^{m-1} \alpha_{j} Z_{j} \mid \alpha_{j} \in[-1 ; 1]\right.$ for all $j=$ $0,1, \cdots, m-1\}$. For all irreducible ${ }^{t} Q$ - invariant subspace $F$ of $\mathbb{R}^{m}$, put $\delta_{F}^{\prime}=\max _{x \in \mathcal{C}^{\prime}}\left\|\pi_{F}(x)\right\|, \quad \lambda_{F}$ the absolute value of the eigenvalue of ${ }^{t} Q$ on $F$ and $c_{F}^{\prime}=\lfloor\beta\rfloor \frac{\delta_{F}^{\prime}}{1-\lambda_{F}^{m}}$. Denote by $\Omega_{F}^{\prime}$ the closed interval centred at 0 in $F$ of length $2 c_{F}^{\prime}$ if $\operatorname{dim} F=1$, resp. the closed disc centred at 0 in $F$ of radius $c_{F}^{\prime}$ if $\operatorname{dim} F=2$. Let $\Omega^{\prime}=\oplus_{F} \Omega_{F}^{\prime}$. We will denote by $\mathcal{T}_{I, R}:=\left\{x \in \mathbb{R}^{m} \mid p_{2}(x) \in\lfloor\beta\rfloor^{-1} R \Omega^{\prime}, \pi_{B}(x) \cdot u_{B} \in\|B\|^{-1} I\right\}$ the 
slice of the band defined by $\lfloor\beta\rfloor^{-1} R \Omega^{\prime}$ in the internal space, extended by symmetrization with respect to $\lfloor\beta\rfloor^{-1} R \Omega$ (compare the definitions of $\Omega^{\prime}$ and $\Omega$ in Theorem 3.6), of axis the expanding line $\mathbb{R} u$. Let $F_{R}:=$ $\left\{\operatorname{frac}(z)\left|z=a_{k} \beta^{k}+a_{k-1} \beta^{k-1}+\cdots+a_{1} \beta+a_{0}, a_{i} \in \mathbb{Z},\right| a_{i} \mid \leq R\right\} \subset[0,1)$.

Lemma 4.6. The set $\left\{\|B\| \pi_{B}(g) \cdot u_{B} \mid g \in \mathcal{T}_{[0,1), R+\lfloor\beta\rfloor} \cap \mathbb{Z}^{m}\right\}$ is a finite subset of $\mathbb{Z}[\beta] \cap[0,1)$ and the following inclusion holds: $F_{R} \subset\left\{\|B\| \pi_{B}(g)\right.$. $\left.u_{B} \mid g \in \mathcal{T}_{[0,1), R+\lfloor\beta\rfloor} \cap \mathbb{Z}^{m}\right\}$.

Proof. The finiteness of the set is obvious: it is a discrete set in a subset of $\mathbb{R}^{m}$ having compact closure. The inclusion relation is a reformulation of Lemma 2.1 in [Bu]. Let us briefly recall the proof. Let $z=\sum_{j=0}^{k} a_{j} \beta^{j}$ with $a_{i} \in \mathbb{Z},\left|a_{i}\right| \leq R$. We have also $z=\sum_{j=-\infty}^{k} x_{j} \beta^{j}$ as $\beta$ - expansion of $z$. Therefore $z-\operatorname{int}(z)=\sum_{i=0}^{k} a_{j} \beta^{k}-\sum_{j=0}^{k} x_{j} \beta^{j}$. Since $0 \leq x_{j} \leq\lfloor\beta\rfloor$ and $\left|a_{i}\right| \leq R, \operatorname{frac}(z) \in[0,1)$ is a polynomial in $\beta$, the coefficients of which have their absolute values bounded by $R+\lfloor\beta\rfloor$. Here the coefficients may be negative or positive. This is why we have introduced $\mathcal{C}^{\prime}$ instead of $\mathcal{C}$. We deduce the result in a similar way as in the proof of Theorem 3.6 for the computation of the upper bound $c_{F}$, except that now it is with $\Omega^{\prime}, c_{F}^{\prime}$ and the fact that the absolute value of the digits is less than $R+\lfloor\beta\rfloor$; this obliges to multiply $\Omega^{\prime}$ by the factor $(R+\lfloor\beta\rfloor) /\lfloor\beta\rfloor$. The set $F_{R}$ is finite (Lemma 6.6 in [So]), and (Proposition 3.4) is in one-to-one correspondence with a subset of the finite point set $\mathcal{T}_{[0,1), R+\lfloor\beta\rfloor}$. We deduce the claim.

Let

$L_{I, R}:=\left\lfloor\min \left\{\left[\ln \left(\beta^{(i-1)^{-1}}\right)\right]^{-1} \ln \left(\left(\sum_{k=0}^{m-1}\left(\beta^{(i-1)}\right)^{2 k}\right)^{1 / 2} \psi_{I, R+\lfloor\beta\rfloor}\right)\right\}\right\rfloor$ where the minimum is taken over the real positive embeddings of $\mathbb{Q}(\beta)(i=$ $1,2, \ldots, s$ and $\left.\beta^{(i-1)}>0\right)$ and where $\psi_{I, R}:=\max \left\{\|y\| \mid y \in \mathcal{T}_{I, R}\right\}$. Let us consider an element $z \in F_{R}$. Its $\beta$ - expansion: $\sum_{j=1}^{+\infty} z_{-j} \beta^{-j}$ is eventually periodic $[\mathrm{Be}][\mathrm{Sch}]$ and therefore can be written $\sum_{j=1}^{k_{0}(z)} z_{-j} \beta^{-j}+$ $\sum_{k=0}^{+\infty} \quad \sum_{j=k_{0}(z)+k r(z)+1}^{k_{0}(z)+(k+1) r(z)} z_{-j} \beta^{-j}$ where the integers $k_{0}(z), r(z) \geq 1$ are minimal. We will denote by $J_{R}=\max \left\{k_{0}(z) \mid z \in F_{R}\right\}$ the maximal preperiod of the $\beta$ - expansions of the elements of $F_{R}$. An upper bound of $J_{R}$ will be computed below.

Theorem 4.7. (i) For all $x, y \in \mathbb{Z}_{\beta}^{+}$such that $x+y$ has a finite $\beta$-expansion the following relation holds: $x+y \in \beta^{-L} \mathbb{Z}_{\beta}^{+}$where $L:=\min \left\{L_{[0,1), 2\lfloor\beta\rfloor}, J_{2\lfloor\beta\rfloor}\right\} ;$ (ii) the following inclusions hold: $\mathbb{Z}_{\beta}^{+}+\mathbb{Z}_{\beta}^{+} \subset$ $\mathbb{Z}_{\beta}^{+}+T, \mathbb{Z}_{\beta}^{+}-\mathbb{Z}_{\beta}^{+} \subset \mathbb{Z}_{\beta}+T^{\prime}$, where $T=\left\{\|B\| \pi_{B}(g) \cdot u_{B} \mid\right.$ $\left.g \in \mathcal{T}_{[0,+1), 3\lfloor\beta\rfloor} \cap \mathbb{Z}^{m}\right\}$ and $T^{\prime}=\left\{\|B\| \pi_{B}(g) \cdot u_{B} \mid g \in \mathcal{T}_{(-1,+1), 2\lfloor\beta\rfloor} \cap \mathbb{Z}^{m}\right\}$. 
Proof. (i) Let $x=x_{k} \beta^{k}+\cdots+x_{0}$ and $y=y_{l} \beta^{l}+\cdots+y_{0}$ denote two elements of $\mathbb{Z}_{\beta}^{+}$. Then $z=x+y$ is of the form $z=a_{j} \beta^{j}+\cdots+$ $a_{0}$ with $0 \leq a_{j} \leq 2\lfloor\beta\rfloor$. Write now the $\beta$ - expansion of $z$ as $z=\sum_{j=1}^{+\infty} z_{-j} \beta^{-j}+\sum_{j=0}^{e} z_{j} \beta^{j}$ and assume it is finite. Then it admits only a $\beta$-expansion up till the term indexed by its preperiod $k_{0}(z)$ and the period has necessarily the form given above with $r(z)=1$ and $z_{-j}=0$ as soon as $j>k_{0}(z)$. Then $\sum_{j=1}^{k_{0}(z)} z_{-j} \beta^{-j}=\left(a_{j} \beta^{j}+\cdots+a_{0}\right)-\left(\sum_{i=0}^{e} z_{i} \beta^{i}\right)$. This means that the fractional part $\sum_{j=1}^{k_{0}(z)} z_{-j} \beta^{-j}$ is a polynomial of the type $\sum_{i=0}^{f} b_{i} \beta^{i}$ with $-\lfloor\beta\rfloor \leq b_{i} \leq 2\lfloor\beta\rfloor$ hence with $\left|b_{i}\right| \leq 2\lfloor\beta\rfloor$. The set $F_{2\lfloor\beta\rfloor}$ is finite (Lemma 4.6 ) and the set of all possible fractional parts of elements of $\mathbb{Z}_{\beta}^{+}$is exactly in one-to-one correspondence with a subset of the finite point set $\mathcal{T}_{[0,1), 3\lfloor\beta\rfloor} \cap \mathbb{Z}^{m}$ of $\mathbb{Z}^{m}$. Therefore, there exists a unique $g_{z}=\sum_{i=0}^{f} b_{i} Z_{i} \in \mathcal{T}_{[0,1), 3\lfloor\beta]} \cap \mathbb{Z}^{m}$ such that $\|B\| \pi_{B}\left(g_{z}\right) \cdot u_{B}=$ $\sum_{i=0}^{f} b_{i} \beta^{i}=\sum_{j=1}^{k_{0}(z)} z_{-j} \beta^{-j}=\operatorname{frac}(z)$. Let us apply the real and complex embeddings of the number field $\mathbb{Q}(\beta)$. It gives: $\sum_{j=1}^{k_{0}(z)} z_{-j}\left(\beta^{(i-1)}\right)^{-j}$ $=\sum_{j=0}^{f} b_{j}\left(\beta^{(i-1)}\right)^{j}$ for all $i=2,3, \ldots, m$. For the real embeddings in particular this implies (Proposition 3.2):

$\pi_{B, i}\left(g_{z}\right)=\pi_{B, i}\left(\sum_{j=0}^{f} b_{j} Z_{j}\right)=\frac{\sum_{j=0}^{f} b_{j}\left(\beta^{(i-1)}\right)^{j}}{\left\|X_{i}\right\|} u_{B, i}=\frac{\sum_{j=1}^{k_{0}(z)} z_{-j}\left(\beta^{(i-1)}\right)^{-j}}{\left\|X_{i}\right\|} u_{B, i}$

for all $i=1,2, \cdots, s$ with all $z_{-j} \geq 0$. The case of real embeddings will provide a direct computation of the first upper bound $L_{[0 ; 1), 2[\beta]}$ of the preperiod and merits to be isolated. Indeed, since in this case $0<\beta^{(i-1)}<$ 1 for all $i \in\{2,3, \cdots, s\}$, with $s$ assumed $\geq 2$, and that all the digits $z_{-j}$ are positive, we necessarily have: $\left\|X_{i}\right\|^{-1}\left(\beta^{(i-1)}\right)^{-j} \geq \psi_{[0,1), 3\lfloor\beta\rfloor}$ as soon as $j$ is large enough. Recall that $\left\|X_{i}\right\|=\left(\sum_{k=0}^{m-1}\left(\beta^{(i-1)}\right)^{2 k}\right)^{1 / 2}$. With the definition of $L_{[0 ; 1), 2\lfloor\beta\rfloor}$, this implies that the sum of the positive terms $\sum_{j=1}^{k_{0}(z)} z_{-j}\left(\beta^{(i-1)}\right)^{-j}$ cannot contain any term indexed by $-j$ with $j>$ $L_{[0 ; 1), 2\lfloor\beta\rfloor}$. Hence, $k_{0}(z) \leq L_{[0 ; 1), 2\lfloor\beta\rfloor}$. As for the negative real embeddings and the complex embeddings they will provide the second upper bound of the preperiod by the computation of $J_{2\lfloor\beta\rfloor}$ : indeed, its calculation gives an upper bound of the number of terms $k_{0}(z)$ in the fractional part of $z$, hence, after reducing $\operatorname{frac}(z)$ to the same denominator, which will be $\beta^{k_{0}(z)}$, we immediately get the result; (ii) (This is reformulation of Theorem 2.4 in $[\mathrm{Bu}])$ First, we have $F_{\lfloor\beta\rfloor} \subset F_{2\lfloor\beta\rfloor}$, second $\mathbb{Z}_{\beta}^{+}+\mathbb{Z}_{\beta}^{+} \subset \mathbb{Z}_{\beta}^{+}+F_{2\lfloor\beta\rfloor}$, $\mathbb{Z}_{\beta}^{+}-\mathbb{Z}_{\beta}^{+} \subset \mathbb{Z}_{\beta}+\left(F_{\lfloor\beta\rfloor} \cup-F_{[\beta]}\right)$. Since $\Omega^{\prime}$ is invariant by inversion and that 
$F_{\lfloor\beta\rfloor} \cup-F_{\lfloor\beta\rfloor} \subset\left\{\|B\| \pi_{B}(g) \cdot u_{B} \mid g \in \mathcal{T}_{[0,+1), 2\lfloor\beta\rfloor} \cap \mathbb{Z}^{m}\right\} \cup\left\{\|B\| \pi_{B}(g) \cdot u_{B} \mid g \in\right.$ $\left.\mathcal{T}_{(-1,0], 2\lfloor\beta\rfloor} \cap \mathbb{Z}^{m}\right\}=\left\{\|B\| \pi_{B}(g) \cdot u_{B} \mid g \in \mathcal{T}_{(-1,+1), 2\lfloor\beta\rfloor} \cap \mathbb{Z}^{m}\right\}$ (Lemma 4.6), we deduce the claim.

Corollary 4.8. Let $q=\min \left\{L_{(-1,+1), 2\lfloor\beta\rfloor}, J_{2\lfloor\beta\rfloor}\right\}$. Then, for all $x, y \in$ $\mathbb{Z}_{\beta}$ such that $x+y$ and $x-y$ have finite $\beta$-expansions, the following relations hold: $x+y$ (resp. $x-y) \in \beta^{-q} \mathbb{Z}_{\beta}$.

Proof. Indeed, $T^{\prime} \subset T \cup(-T)$. Hence $\mathbb{Z}_{\beta}+\mathbb{Z}_{\beta} \subset \mathbb{Z}_{\beta}+(T \cup(-T))$. Since $T \cup(-T)=\left\{\|B\| \pi_{B}(g) \cdot u_{B} \mid g \in \mathcal{T}_{(-1,+1), 3\lfloor\beta\rfloor} \cap \mathbb{Z}^{m}\right\}$, we deduce the exponent $q$ from the definition of $L_{I, R}$ and from Theorem 4.7.

Computation of an upper bound of the maximal preperiod $J_{R} \cdot-$ We will use the case (ii) of cut-and-project scheme in Theorem 3.5. Let $\left\{Z_{-j}\right\}_{j \geq 0}$ be the sequence of vectors defined by $Z_{0}=\left({ }^{t} Q\right)^{j} Z_{-j}$. We denote as usual the algebraic norm of $\beta$ by $N(\beta)=N_{\mathbb{Q}(\beta) / \mathbb{Q}}(\beta)=\prod_{i=0}^{m-1} \beta^{(i)}$. Recall that $a_{0}=(-1)^{m-1} N(\beta)$.

Lemma 4.9. (i) The following limit holds: $\lim _{j \rightarrow+\infty}\left\|Z_{-j}\right\|=+\infty$; (ii) for all $j \in \mathbb{N}, Z_{-j} \in N(\beta)^{-j} \mathbb{Z}^{m}$. In particular, if $\beta$ is a unit of the number field $\mathbb{Q}(\beta)$, then all the elements $Z_{-j}$ belong to $\mathbb{Z}^{m}$.

Proof. (i) Since $\left|\beta^{(i)}\right|^{-1}>1$ for all $i=1,2, \ldots, m-1$, the inverse operator $\left({ }^{t} Q\right)^{-1}$ acts as a dilation by a factor of modulus strictly greater than one on each ${ }^{t} Q$-invariant subspace $F$ in $\mathbb{R}^{m}$ except $\mathbb{R} u$ : all the non-zero components of the vector $Z_{-j}$ (which never belongs to $\mathbb{R} u$ ) in the system $\left\{V_{i}\right\}_{i=2,3, \cdots, m}$ diverge when $j$ tends to infinity, hence the claim. (ii) Solving the equation $Z_{0}=\left({ }^{t} Q\right)^{1} Z_{-1}$ shows that $Z_{-1}$ can be written $Z_{-1}=-a_{0}^{-1}\left(a_{1} Z_{0}+a_{2} Z_{1}+\cdots+a_{m-1} Z_{m-2}-Z_{m-1}\right) \in N(\beta)^{-1} \mathbb{Z}^{m}$. Since by construction we have $Z_{j}=\left({ }^{t} Q\right)^{-1}\left(Z_{j+1}\right)$ for all $j \in \mathbb{Z}$, applying $\left({ }^{t} Q\right)^{-1}$ to the last equality clearly gives $Z_{-2} \in N(\beta)^{-2} \mathbb{Z}^{m}$ and, by induction $Z_{-h} \in N(\beta)^{-h} \mathbb{Z}^{m}$ for all $h \geq 0$. Now it is classical that $\beta$ is a unit of $\mathbb{Q}(\beta)$ if and only if $N(\beta)= \pm 1$ establishing the result.

Theorem 4.10. Denote by

$$
\begin{aligned}
& \mathcal{B}_{R}=\left\{x \in \mathbb{R}^{m} \mid\left\|\pi_{B, i}(x)\right\| \leq \frac{\psi_{[0 ; 1), R+\lfloor\beta\rfloor}\left(1-\left|\beta^{(i-1)}\right|^{m}\right)+\lfloor\beta\rfloor}{\left(\sum_{k=0}^{m-1}\left|\beta^{(i-1)}\right|^{2 k}\right)^{1 / 2}\left(1-\left|\beta^{(i-1)}\right|\right)},\right. \\
& i=2,3, \cdots, m\}
\end{aligned}
$$

the cylinder (band) of axis the expanding line $\mathbb{R} u$ and $\mathcal{V}_{R}=\{x \in$ $\left.\mathcal{B}_{R} \mid\|B\| \pi_{B}(x) \cdot u_{B} \in[0,1)\right\}$ the slice of the band $\mathcal{B}_{R}$. Then this slice is such that $J_{R} \leq \#\left(\mathcal{V}_{R} \cap N(\beta)^{-m} \mathbb{Z}^{m}\right)$. 
Proof. Each element $\alpha \in F_{R}$ can be written $\alpha=\sum_{i=0}^{m-1} p_{i} \beta^{i}$ with $p_{i} \in \mathbb{Z}$ and $\sum_{i=0}^{m-1} p_{i} Z_{i} \in \mathcal{T}_{[0,1), R+\lfloor\beta\rfloor} \cap \mathbb{Z}^{m}$ (Lemma 4.6). Thus, $\left|p_{i}\right| \leq$ $\psi_{[0,1), R+\lfloor\beta\rfloor}$ for all $i=0,1, \cdots, m-1$. Now ([Sch] and section 2), the following equality holds for all $n \geq 0$ :

$$
T^{n}(\alpha)=\beta^{n} \cdot\left(\alpha-\sum_{k=0}^{n} \epsilon_{k}(\alpha) \beta^{-k}\right)=\sum_{k=1}^{m} r_{k}^{(n)} \beta^{-k}
$$

where $\left(\epsilon_{k}(\alpha)\right)_{k \geq 0}$ is the sequence of digits of the Rényi $\beta$-expansion of $\alpha$ and $\left(r_{1}^{(n)}, r_{2}^{(n)}, \cdots, r_{m}^{(n)}\right) \in \mathbb{Z}^{m}$. Recall that $\epsilon_{0}(\alpha)=\lfloor\alpha\rfloor=0$. The real and complex embeddings of the number field $\mathbb{Q}(\beta)$ applied to $T^{n}(\alpha)$ provide the $m$ equalities, with $j=1,2, \cdots, m$ :

$$
\begin{aligned}
\left(\beta^{(j-1)}\right)^{n} \cdot\left(\sum_{i=0}^{m-1} p_{i}\left(\beta^{(j-1)}\right)^{i}-\sum_{k=1}^{n} \epsilon_{k}(\alpha)\left(\beta^{(j-1)}\right)^{-k}\right) & = \\
& \sum_{k=1}^{m} r_{k}^{(n)}\left(\beta^{(j-1)}\right)^{-k} .
\end{aligned}
$$

We deduce that

$$
\begin{aligned}
\left(\sum_{k=0}^{m-1}\left|\beta^{(i-1)}\right|^{2 k}\right)^{1 / 2} & \left\|\pi_{B, i}\left(\sum_{k=1}^{m} r_{k}^{(n)}\left(\beta^{(j-1)}\right)^{-k}\right)\right\| \\
& =\left|\sum_{k=1}^{m} r_{k}^{(n)}\left(\beta^{(j-1)}\right)^{-k}\right| \\
& \leq \sum_{i=0}^{m-1}\left|p_{i}\right|\left|\beta^{(j-1)}\right|^{n+i}+\lfloor\beta\rfloor \sum_{k=0}^{n}\left|\beta^{(j-1)}\right|^{k} \\
& \leq \frac{1}{1-\left|\beta^{(j-1)}\right|}\left[\psi_{[0,1), R+\lfloor\beta\rfloor}\left(1-\left|\beta^{(j-1)}\right|^{m}\right)+\lfloor\beta\rfloor\right]
\end{aligned}
$$

for all $n \geq 0, j=2,3, \cdots, m$ with $0 \leq \sum_{k=1}^{m} r_{k}^{(n)} \beta^{-k}<1$. From Proposition 3.2 and Lemma 4.9 the element $\sum_{k=1}^{m} r_{k}^{(n)} \beta^{-k}$ can be uniquely lifted up to the element $\sum_{k=1}^{m} r_{k}^{(n)} Z_{-k} \in N(\beta)^{-m} \mathbb{Z}^{m}$. Its projections by the projection mappings $\pi_{B, i}, i=2,3, \cdots, m$ to the $Q$-invariant subspaces of $\mathbb{R}^{m}$ are bounded by constants which are independant of $n$. The restriction of the lifting of the operator $T$ to $\mathcal{V}_{R} \cap N(\beta)^{-m} \mathbb{Z}^{m}$ has self-avoiding orbits (to have a preperiod) whose length is necessarily smaller than the number of available points in the volume $\mathcal{V}_{R}$. We deduce the upper bound $\#\left(\mathcal{V}_{R} \cap N(\beta)^{-m} \mathbb{Z}^{m}\right)$ of $J_{R}$. 
Appendix.- Classification of Delone sets. We will say that a subset $\Lambda$ of $\mathbb{R}^{n}$ is (i) uniformly discrete if there exists $r>0$ such that $\|x-y\| \geq r$ for all $x, y \in \Lambda, x \neq y$, (ii) relatively dense if there exists $R>0$ such that, for all $z \in \mathbb{R}^{n}$, there exists $\lambda \in \Lambda$ such that the ball $B(z, R)$ contains $\lambda$, (iii) a Delone set if it is relatively dense and uniformly discrete. Delone sets are basic objects for mathematical quasicrystals [La2] [MVG].

Definition 4.1. A cut-and-project scheme consists of a direct product $E \times D$, where $E$ and $D$ are Euclidean spaces of finite dimension, and a lattice $L$ in $E \times D$ so that, with respect to the natural projections $p_{1}: E \times D \rightarrow E, p_{2}: E \times D \rightarrow D:(\mathrm{i}) \quad p_{1}$ restricted to $L$ is one-to-one onto its image $p_{1}(L)$, (ii) $p_{2}(L)$ is dense in $D$. We will denote by $*$ the following operation: $*:=p_{2} \circ\left(p_{1_{\left.\right|_{L}}}\right)^{-1}: p_{1}(L) \rightarrow D$.

Definition 4.2. A subset $\Lambda$ of a finite dimensional Euclidean space $E$ is a model set (also called a cut-and-project set) if there exist a cut-and-project scheme $(E \times D, L)$ and a subset $\Omega$ of $D$ with nonempty interior and compact closure such that $\Lambda=\Lambda(\Omega)=\left\{p_{1}(l) \mid l \in L, p_{2}(l) \in\right.$ $\Omega\}$, equivalently $=\left\{v \in p_{1}(L) \mid v^{*} \in \Omega\right\}$. The set $\Omega$ is called acceptance window.

Meyer sets were introduced in [Mey]. By definition, we will say that $\Lambda$, assumed to be a relatively dense subset of $\mathbb{R}^{n}$, is a Meyer set of $\mathbb{R}^{n}$ if it is a subset of a model set. Other equivalent definitions can be found in [Mo] or [Mey]. For instance, $\Lambda$ is a Meyer set if and only if it is a Delone set and there exists a finite set $F$ such that $\Lambda-\Lambda \subset \Lambda+F$; or if and only if it is relatively dense and $\Lambda-\Lambda$ is uniformly discrete. The above definition shows that the class of Meyer sets of $\mathbb{R}^{n}$ contains the class of model sets of $\mathbb{R}^{n}$.

Theorem 4.11. (Meyer [Mey]) Let $\Lambda$ be a Delone set in $\mathbb{R}^{n}$ such that $\eta \Lambda \subset \Lambda$ for a real number $\eta>1$. If $\Lambda$ is a Meyer set, then $\eta$ is a Pisot or a Salem number.

Definition 4.3. A Delone set $\Lambda$ is said to be finitely generated if $\mathbb{Z}[\Lambda-\Lambda]$ is finitely generated. A Delone set $\Lambda$ is said to be of finite type if $\Lambda-\Lambda$ is such that its intersection with any closed ball of $\mathbb{R}^{n}$ is a finite set.

The class of finitely generated Delone sets of $\mathbb{R}^{n}$ is strictly larger than the class of Delone set of finite type of $\mathbb{R}^{n}$, which is itself larger than the class of Meyer sets of $\mathbb{R}^{n}$ [La] [La1].

Theorem 4.12. (Lagarias [La]) Let $\Lambda$ be a Delone set in $\mathbb{R}^{n}$ such that $\eta \Lambda \subset \Lambda$ for a real number $\eta>1$. The following assertions hold: (i) If $\Lambda$ is finitely generated, then $\eta$ is an algebraic integer. If the rank of $\mathbb{Z}[\Lambda]$ is $s$, then the degree of $\eta$ divides $s$, (ii) If $\Lambda$ is a Delone set of finite type, then $\eta$ is a Perron number or is a Lind number. 
Although $\mathbb{Z}_{\beta}$ is associated with two canonical cut-and-project schemes when $\beta$ is a non-integer Perron number, the converse of the assertion (ii) of Theorem 4.12 seems to be an open problem. It is at least already related to the question Q1' of the introduction and to various arithmetical and dynamical problems [ABEI].

Acknowledgements. - We are indebted to Christiane Frougny, Valérie Berthé, Pierre Arnoux and a referee for very useful and valuable comments and discussions.

\section{References}

[Ak] S. Akryama, Cubic Pisot units with finite $\beta$-expansions. Algebraic number theory and Diophantine analysis, (Graz, 1998), de Gruyter, Berlin, (2000), 11-26.

[Ak1] S. AkrYama, Pisot numbers and greedy algorithm. Number Theory, (Eger, 1996), de Gruyter, Berlin, (1998), 9-21.

[ABEI] P. ARNOUX, V. BERThÉ, H. EI and S. ITO, Tilings, quasicrystals, discrete planes, generalized substitutions and multidimensional continued fractions. Disc. Math. and Theor. Comp. Sci. Proc. AA (DM-CCG), (2001), 59-78.

[AI] P. Arnoux and S. ITO, Pisot substitutions and Rauzy fractals. Bull. Belg. Math. Soc. 8 (2001), 181-207.

[Be] A. Bertrand, Développements en base de Pisot et répartition modulo 1. C. R. Acad. Sc. Paris, Série A, t. 285 (1977), 419-421.

[Be1] A. BeRTRAND - MATHIS, Comment écrire les nombres entiers dans une base qui n'est pas entière. Acta Math. Hung., 54, (3-4) (1989), 237-241.

[Be2] A. Bertrand - MATHIS, Développement en base $\theta$, Répartition modulo un de la suite $\left(x \theta^{n}\right)_{n \geq 0}$. Langages codes et $\theta$ - shift, Bull. Soc. Math. France, 114 (1986), 271-323.

[Be3] A. Bertrand - Mathis, Nombres de Perron et questions de rationnalité. Séminaire d'Analyse, Université de Clermont II, Année 1988/89, Exposé 08; A. BerTrand Mathis, Nombres de Perron et problèmes de rationnalité. Astérisque, 198-199-200 (1991), 67-76.

[Be4] A. BERTRAND - MATHIS, Questions diverses relatives aux systèmes codés: applications au $\theta$-shift. Preprint.

[Bl] F. Blanchard, $\beta$-expansions and Symbolic Dynamics. Theor. Comp. Sci. 65 (1989), 131-141.

[Bu] Č. Burdík, Ch. Frougny, J.-P. Gazeau and R. Krejcar, Beta-integers as natural counting systems for quasicrystals. J. Phys. A: Math. Gen. 31 (1998), 6449-6472.

[Fro] C. FrougnY, Number representation and finite automata. London Math. Soc. Lecture Note Ser. 279 (2000), 207-228.

[Fro1] Ch. Frougny and B. SolomyaK, Finite beta-expansions. Ergod. Theor. Dynam. Syst. 12 (1992), 713-723.

[Gaz] J. P. GazeAU, Pisot-Cyclotomic Integers for Quasicrystals. The Mathematics of LongRange Aperiodic Order, Ed. By R.V. MoodY, Kluwer Academic Publishers, (1997), 175-198.

[HS] M. W. Hirsch and S. Smale, Differential Equations. Dynamical Systems and Linear Algebra, Academic Press, New York, (1974).

[IK] S. Ito and M. Kimura, On the Rauzy fractal. Japan J. Indust. Appl. Math. 8 (1991), 461-486.

[IS] S. Ito and Y. SANo, On periodic $\beta$ - expansions of Pisot numbers and Rauzy fractals. Osaka J. Math. 38 (2001), 349-368.

[La] J. Lagarias, Geometric Models for Quasicrystals I. Delone Sets of Finite Type. Discrete Comput. Geom. 21 no 2 (1999), 161-191. 
[La1] J.C. LAGARIAS, Geometrical models for quasicrystals. II. Local rules under isometry. Disc. and Comp. Geom., 21 no 3 (1999), 345-372.

[La2] J.C. LAGARIAS, Mathematical Quasicrystals and the problem of diffraction. Directions in Mathematical Physics, CRM Monograph Series, Vol 13, Ed. M. BAAKE and R.V. MoODY, (2000), 61-94.

[Li] D. LIND, The entropies of topological Markov shifts and a related class of algebraic integers. Ergod. Th. \& Dynam. Sys. 4 (1984), 283-300.

[Me] A. MEssaOUd, Propriétés arithmétiques et dynamiques du fractal de Rauzy. J. Théor. Nombres Bordeaux, 10 (1998), 135-162.

[Me1] A. MESSAOUd, Frontière du fractal de Rauzy et système de numérotation complexe Acta Arith. 95 (2000), 195-224.

[Mey] Y. MEYER, Algebraic Numbers and Harmonic Analysis. North-Holland (1972).

[Mi] H. MINC, Nonnegative matrices. John Wiley and Sons, New York (1988).

[Mo] R.V. Moody, Meyer sets and their duals The Mathematics of Long-Range Aperiodic Order, Ed. By R.V. Moody, Kluwer Academic Publishers, (1997), 403-441.

[MVG] G. MURAz and J.-L. Verger-GAUGRY, On lower bounds of the density of packings of equal spheres of $\mathbb{R}^{n}$. Institut Fourier, preprint $\mathrm{n}^{\circ} \mathbf{5 8 0}(2003)$.

[Pa] W. PARRY, On the $\beta$ - expansions of real numbers. Acta Math. Acad. Sci. Hung. 11 (1960), 401-416.

[PF] N. PytheAs FOGG, Substitutions in dynamics, arithmetics and combinatorics. Lect. Notes Math. 1794, Springer-Verlag, (2003).

[Ra] G. RAUZY, Nombres algébriques et substitutions. Bull. Soc. Math. France, 110 (1982), 147-178.

[Re] A. RENYI, Representations for real numbers and their ergodic properties. Acta Math. Acad. Sci. Hung. 8 (1957), 477-493.

[Ru] D. Ruelle, Statistical Mechanics; Rigorous results. Benjamin, New York, (1969).

[Sc] J. Schmeling, Symbolic dynamics for $\beta$ - shifts and self-normal numbers. Ergod. Th. \& Dynam. Sys. 17 (1997), 675-694.

[Sch] K. SchмIDT, On periodic expansions of Pisot numbers and Salem numbers. Bull. London Math. Soc. 12 (1980), 269-278.

[So] B. SolOmYAK, Dynamics of Self-Similar Tilings. Ergod. Th. \& Dynam. Sys. 17 (1997), 695-738.

[Th] W. ThuRston Groups, tilings and finite state automata. Preprint (1989).

Jean-Pierre GAZEAU

LPTMC

Université Paris 7 Denis Diderot

mailbox 7020

2 place Jussieu

75251 Paris Cedex 05, France

E-mail : gazeau@ccr.jussieu.fr

Jean-Louis Verger-GaUGRY

Institut Fourier

UJF Grenoble

UFR de Mathématiques

CNRS UMR 5582

BP 74 - Domaine Universitaire

38402 Saint Martin d'Hères, France

E-mail : jlverger@ujf-grenoble.fr 